PALEO

Revue d'archéologie préhistorique

29 | 2018

Varia

\title{
Le Périgordien, quelle erreur !
}

The Perigordian, What a mistake!

\section{Damien Pesesse}

\section{OpenEdition}

Journals

Édition électronique

URL : http://journals.openedition.org/paleo/4094

DOI : $10.4000 /$ paleo.4094

ISSN : 2101-0420

Éditeur

SAMRA

\section{Édition imprimée}

Date de publication : 30 décembre 2018

Pagination : 179-199

ISSN : 1145-3370

\section{Référence électronique}

Damien Pesesse, «Le Périgordien, quelle erreur! », PALEO [En ligne], 29 | 2018, mis en ligne le 15

janvier 2020, consulté le 07 juillet 2020. URL : http://journals.openedition.org/paleo/4094 ; DOI https://doi.org/10.4000/paleo.4094

\section{(c) (i) (9)}

PALEO est mis à disposition selon les termes de la licence Creative Commons Attribution - Pas d'Utilisation Commerciale - Pas de Modification 4.0 International. 


\title{
Le Périgordien, quelle erreur !
}

\author{
Damien PESESSE ${ }^{(a)}$
}

Résumé : Le Périgordien est abandonné ; ce taxon a perdu toute légitimité scientifique. Qu'en faire ? Ce bilan bibliographique contribue à ouvrir le débat en faisant le point sur la documentation bibliographique dans une période comprise essentiellement entre 1930 et 1970. Après un long travail historiographique, ce fossile est interrogé en fonction du « principe de symétrie » de manière à ne pas se placer du côté des vainqueurs, l'Aurignacien et le Gravettien. Cela permet de comprendre certains des modes de raisonnements déployés par les chercheurs durant cette période. Le Périgordien peut ainsi être considéré comme une manière singulière de concilier succession stratigraphique et rupture technique. En morcelant le temps et l'espace, il contribue à redonner de la continuité à la succession stratigraphique que les fouilles successives questionnent régulièrement. Durant le Périgordien, les nouvelles fouilles sont en effet à l'origine des modifications régulières de ce modèle. Les concepts, en partie hérités de $\mathrm{H}$. Breuil, eux ne sont pas questionnés. Les sites archéologiques occupent alors une place tout à fait singulière car ils deviennent la condition privilégiée, voire unique, pour que puisse s'opérer ce qui est perçu comme un progrès scientifique par les acteurs de ce débat. Cette interprétation interroge directement notre manière de situer notre objectivité. II semble que celle-ci soit essentiellement transférée aux sites et aux objets. Cela explique notamment la perduration de certains paradigmes qui restent dans l'ombre de ces débats organisés par les attributions chronologiques ou la lecture du mobilier. Plusieurs possibilités s'offrent alors à nous : soit le Périgordien est une erreur et notre mode d'objectivation n'est pas à réévaluer ; soit le Périgordien n'est pas une erreur et il serait temps de renégocier la confiance que nous accordons aux objets.

Mots-clés : Paléolithique supérieur, Sériation, Historiographie, Collectifs hybrides, Principe de symétrie.

Abstract: The Perigordian, What a mistake ! Perigordian is abandoned; this taxon lost all scientific legitimacy. What is to be done? This bibliographical report contributes to opening the debate by reviewing the bibliographical documentation for a period essentially between 1930 and 1970. After a long historiographical work, this fossil is questioned according to the "symmetry principle" by means of not taking position on the "winning side", the Aurignacian and the Gravettian. This shows an understanding of some of the ways of reasoning deployed by the researchers during this period. Perigordian can be so considered as a singular way of reconciling stratigraphical succession and technical breach. By splitting time and space, it contributes to restoring a continuity in the stratigraphical succession that the successive excavations question regularly. During the Perigordian era, the new excavations are indeed at the origin of regular changes of this model. The concepts, partially inherited from $\mathrm{H}$. Breuil, are not questioned. Archeological sites therefore occupy a singular place because they become the privileged, or even unique condition, so that what is perceived as a scientific progress by the actors of this debate can take place. This interpretation questions directly our way of placing our objectivity. It seems that it is mainly transferred to sites and to objects. It explains in particular the continuation of certain paradigms which stay in the shadows of these debates organized by the chronological attributions or the reading of the artifacts. Several possibilities offer themselves then to us: either the Perigordian is a mistake and our way of objectification do not need to be revalued; or that the Perigordian is not a mistake and it would be time to rethink the trust which we grant to objects.

Key-words: Upper Palaeolithic, seriation, historiography, hybrid collectives, symmetry principle.

(a) UMR 6566 - Creaah, Université Rennes 2 - damien.pesesse@univ-rennes2.fr 


\section{Introduction}

Qui s'intéresse au Périgordien ? À en croire la quantité de références abordant la question, ce ne serait pas un sujet, trop loin des tonalités épiques de la bataille aurignacienne, qui a depuis longtemps intégré le temps du mythe de la cosmogonie préhistorienne, trop loin des hautes couleurs des héros de la Préhistoire. Tout se passe comme si l'on devait faire comme $\mathrm{H}$. Breuil et rayer de notre biographie commune celui qui fut d'abord l'instituteur des Eyzies. Seuls quelques experts périgourdins résistent, nécessairement confrontés à son important travail de terrain. Mais même si l'on s'intéresse aux fouilles, à la vie, à l'histoire de D. Peyrony, on ne s'intéresse pas nécessairement au Périgordien, ce fossile bien rangé au fond des bibliothèques.

Formulé par D. Peyrony en 1933, le Périgordien rencontra pourtant un franc succès au sein de la communauté des préhistoriens. La rapidité avec laquelle ce système fut adopté comme sa longévité en attestent. Pendant un demisiècle, le Périgordien constitue le principal système de sériation des industries du Paléolithique supérieur ancien. Aussi étonnant que le succès du Périgordien, le désintérêt actuel pour ce sujet est tout aussi éloquent. D'ailleurs, qu'est-ce que le Périgordien ? Une hypothèse, un modèle, une théorie ? Nous tenterons de le définir. II sera décrit ici comme un modèle dans la partie historiographique, modèle interprétatif et modèle chronologique. II sera parfois présenté comme un taxon, au sens d'une unité élémentaire d'un système de classification, sans lui donner de connotation biologique (Travers 1981).

Des travaux récents ont documenté l'évolution de la sériation du Périgordien (Sackett 1991 ; Bosselin et Djindjian 1994 ; Djindjian et Bosselin 1994 ; Klaric 2003 ; Goutas 2004 ; Bernaldo de Quiros et Neira 2012 ; De la Peña Alonso 2009, 2012). On se penchera plus particulièrement sur les éléments qui ont conduit D. Peyrony à revenir sur la chronologie proposée par $H$. Breuil, en présentant l'évolution des données, principalement à partir des gisements centraux de son modèle, dont La Ferrassie. Les arguments proviennent donc principalement de la plume et de la truelle de D. Peyrony. Nous verrons ensuite comme le Périgordien a survécu à la mort de son père.

Le lieu n'est pas de présenter ici l'ensemble du processus de construction conduisant à la sériation du Paléolithique supérieur telle que nous la connaissons aujourd'hui. L'origine de la reconnaissance de l'Aurignacien et la question aurignacienne ont fait l'objet de travaux spécifiques (voir par exemple Dubois 2006 ; Dubois et Bon 2006 et bibliographie associée). Rétrospectivement, cette controverse est redistribuée dans plusieurs dynamiques : le passage de la période à la culture et dans une série d'oppositions : gradualisme et saltationnisme, continuité et rupture, unilinéarité et multiphylétisme (Groenen 1994 ; Guillomet-Malmassari 2012 ; Lippé 2012).

Rappelons qu'à la suite des travaux d'A. Rutot et d'E. Piette, $H$. Breuil poursuit l'idée de l'existence d'une phase présolutréenne. Cette période, l'éburnéen de Brassempouy, observée également à Tarté, Aurignac et Cro-magnon, trouve son expression complète à Solutré qui livre en 1907 aux congressistes d'Autun en stratigraphie l'Aurignacien, le Solutréen et le Magdalénien (Combier et al. 2002 ; Dubois
2006). La bataille qui opposa $\mathrm{H}$. Breuil à Gabriel, puis Adrien de Mortillet, entre 1901 et 1907 modifia profondément l'évolution, mais également la vision des sociétés préhistoriques. L'une des conséquences de la « bataille aurignacienne » fut la reconnaissance de l'Aurignacien (Breuil 1907a et b), subdivisé par la suite en Aurignacien inférieur, moyen et supérieur (Breuil 1913). Une véritable « rupture conceptuelle » au sein de la discipline pourrait être située à ce moment (Coye 2011).

Comment un dissensus s'est progressivement opéré entre H. Breuil et D. Peyrony ? Quelles observations stratigraphiques et techniques sont au centre de ces discussions ? Comment ce modèle s'est-il construit et a évolué ?

Plusieurs éléments questionnent les conditions de cette domination. Tout d'abord, les arguments retenus par $D$. Peyrony puis ses successeurs révèlent une certaine partialité voire des contradictions internes relevées par D. Peyrony lui-même. Ensuite, seulement trois ans après l'énoncé du Périgordien, D. Garrod (1936) propose de différencier le Gravettien. Étonnantes sont également les critiques formulées y compris par certains défenseurs du Périgordien dès les années 1950. Très vite, un décalage important se manifeste entre la véhémence que revêt le débat et la nature des arguments employés. A posteriori, le Périgordien apparaît comme une parenthèse de l'histoire de la recherche, une sorte d'erreur évolutive dans une discipline qui ne pourrait que progresser par une incrémentation progressive des données. II n'y aurait donc rien à apprendre de lui. Le Périgordien était une erreur, aujourd'hui dépassée.

Sur la base d'un recensement bibliographique, une histoire peut être retracée selon une méthodologie un peu obsolète, relatant chronologiquement les évolutions du terme et ses conditions d'emploi. À chaque moment, je tenterai de confronter les arguments retenus à d'autres arguments qu'il était possible de mobiliser en faveur ou contre le modèle Périgordien. Cette mise en œuvre de potentialités déjouera partiellement le caractère relativement linéaire d'un récit chronologique. Les arguments que j'emploierai étaient donc disponibles et parfois utilisés pour critiquer le Périgordien.

Beaucoup de points ne seront pas abordés. Les relations entre acteurs de cette histoire constituent l'un des principaux absents de cette trame narrative. Dans le «Résumé de ma vie », D. Peyrony rend compte de l'importance de ce facteur, notamment dans le cadre des demandes de subventions auprès de l'Association Française pour l'Avancement des Sciences (White et Roussot 2003). On pressent le rôle joué par la biographie de A. Cheynier (Tronel 2015) dans la difficulté de faire émerger le Gravettien ; on sait une partie de l'impact de la personnalité de F. Bordes pour faire perdurer le Périgordien. Une connaissance plus intime de ces acteurs, l'étude de leurs correspondances fourniraient d'autres modes d'explication que les données stratigraphiques ou techniques transmises par la bibliographie. D'ailleurs, tous les acteurs ne pourront être intégrés à cette discussion. Le rôle des institutions, au cœur de nombreuses études historiographiques, n'est pas pris en compte. L'exhaustivité, si cela était possible, n'est donc pas recherchée ici. Cela tient également à une différence de projet, celui de ne pas distinguer a priori le contexte et le 
contenu (Latour 2001). Donc, il ne s'agit pas d'une publication sur l'histoire de la préhistoire entre 1930 et 1970 mais sur un corpus de publications. D'autres travaux se penchent sur ce début du $X X^{\circ}$ siècle qui ne possède plus le " pittoresque et (...) l'étrangeté de la première archéologie préhistorique » et «ne présente pas encore les marques de la modernité » (Coye 2011).

Le corpus étudié comprend 60 références (articles, notes, monographies ou mémoires) portant directement sur le Périgordien et la période antérieure (1905 - 1933). Elles proviennent principalement du Bulletin de la Société Préhistorique française (29), ce qui correspond bien avec le tropisme géographique des publications à cette période (Soulier 2007, p. 58). Les autres revues sont bien moins représentées : l'Association française pour l'Avancement des Sciences (4), le Congrès de Préhistoire française (5), l'Anthropologie (5), le Congrès International d'Anthropologie et d'Archéologie Préhistorique (5). L'objectif de cette publication est d'ouvrir le débat : faire un point bibliographique et historiographique autour du Périgordien pour apporter une base documentaire. Le temps écoulé depuis renvoie déjà suffisamment ce sujet dans un temps exotique pour ne pas l'aborder aujourd'hui. Le recours accru aux citations permettra ainsi de condenser des éléments importants dispersés dans de nombreux articles, permettant plus facilement à chacun de s'approprier cette problématique.

Cette histoire est retracée à partir des arguments employés par les acteurs. Comme il est hors de question de faire leur procès, tout au contraire, cherchons à comprendre les éléments jugés significatifs, les éléments ayant eu valeur de preuve pour nourrir leurs échanges. Le but n'est donc pas de définir ce qu'ils auraient du faire, mais comment ils ont fait.

Le Périgordien est-il une erreur ? Faut-il encore le laisser prendre la poussière au fond des bibliothèques ? Qu'en faire si ce n'est une histoire vieillotte de la discipline, sans actualité aujourd'hui ? Le principe de symétrie (Bloor 1976) nous invite au contraire à ne pas écrire l'histoire du côté des gagnants. C'est dans cette perspective que sera amenée la réflexion finale. Après un compte rendu très commun de l'historiographie, nous essaierons de comprendre pourquoi dans un processus censé être cumulatif, le sens des objets n'est toujours pas stabilisé. Pour cela, il sera nécessaire de s'appuyer sur l'anthropologie symétrique (Latour 1991) qui nous exhorte également à prendre en compte les rôles des non-humains dans l'élaboration de ce modèle. Sites, objets et catégories d'objets deviennent alors les acteurs à part entière. Ils permettent d'expliquer le Périgordien comme un réseau associant humains, objets, sites, conceptions de l'évolution et du changement donnant alors toute la possibilité d'être de ce modèle. Sortir le Périgordien de l'oubli, c'est abandonner un a priori archaïque à son égard, dans la mesure où il nous permet d'interroger notre mode actuel d'objectivation. Cette perspective sera donc évoquée en synthèse. À présent, place pour le Périgordien !

\section{1 - La Ferrassie, la genèse du Périgordien}

Dans ses premières années, D. Peyrony (1869-1954) se trouve en accord avec H. Breuil (1877-1961). La collaboration qui unit les deux hommes au tout début du
$\mathrm{XX}^{\mathrm{e}}$ siècle et particulièrement en ce qui concerne la reconnaissance de l'art aboutit à plusieurs publications communes. Les fouilles de D. Peyrony et de L. Capitan à La Ferrassie participent directement au débat de la reconnaissance de l'Aurignacien pré-solutréen (Breuil 1905) :

« quant à la couche inférieure, sa faune notablement plus ancienne, son outillage très particulier et nettement différent de la couche sus-jacente permettent de l'identifier aux stations similaires que nous désignerons, avec Breuil, par le terme provisoire de présolutréen, dont Cro-Magnon et Gorge d'Enfer constituent de bons exemples » (Capitan et Peyrony 1905).

En 1906 lors du Congrès de Monaco, H. Breuil propose le terme « aurignacien », accompagné par $\mathrm{E}$. Cartailhac et A. Rutot (Breuil 1907a, 1907b ; Cartailhac 1907 ; Rutot 1907). Cette évolution rapide de la nomenclature s'opère également pour la stratigraphie de La Ferrassie :

"La démonstration est aussi nette que possible, puisqu'il est facile de suivre l'évolution industrielle depuis le Moustérien jusqu'au Solutréen, en passant par l'Aurignacien, nettement intercalé entre ces deux industries, aussi clairement caractérisées d'ailleurs par leurs industries spéciales que l'est l'Aurignacien lui-même » (Capitan et Peyrony 1907- p. 188).

À La Ferrassie toujours, il classe " en accord avec l'abbé Breuil » le niveau, situé entre le Moustérien supérieur et l'Aurignacien moyen, à l'Aurignacien inférieur (Peyrony 1922), suite à la publication des « Subdivisions du Paléolithique supérieur » (Breuil 1913) dans laquelle $\mathrm{H}$. Breuil évoque la continuité morphologique des pointes à dos durant l'Aurignacien (fig. 1). Dans ce niveau, les formes moustériennes, les pointes du type de Châtelperron les "lames à gorges » et les grattoirs du «type de Tarté » coexistent. Cette association avait également été rencontrée à Châtelperron, Germolles, la Roche-au-Loup, Haurets et Gargas (Peyrony 1922). Jusque là, leurs travaux abondent dans la même direction.

L'extension de la fouille, au devant de l'abri, va induire une première nuance. Cette couche d'Aurignacien inférieur se subdivise en deux niveaux distincts séparés par une couche stérile. À cette révision stratigraphique correspond également un changement dans le mobilier : les formes moustériennes se trouvent limitées à l'horizon inférieur, les formes aurignaciennes, au niveau supérieur. À ce stade, Peyrony reste prudent :

«De leur examen, il se dégage l'impression que l'une paraît être la dernière phase du Moustérien, tandis que l'autre semble être l'initiale de l'Aurignacien. Pour l'instant, nous ne tirons pas de conclusions, les réservant pour un travail plus étendu; nous nous bornons simplement à présenter les faits observés et nos impressions 》 (Peyrony 1922 - p. 514)

La découverte de « lamelles à bords retouchés, souvent sur la face plane » (Peyrony 1922 - p. 513), dans la couche supérieure, consomme la rupture avec la strate susjacente ; car si des éléments à dos courbes rappellent encore le niveau inférieur, ces lamelles n'ont « plus aucun rapport avec les pièces moustériennes » (Peyrony 1922 p. 513). 


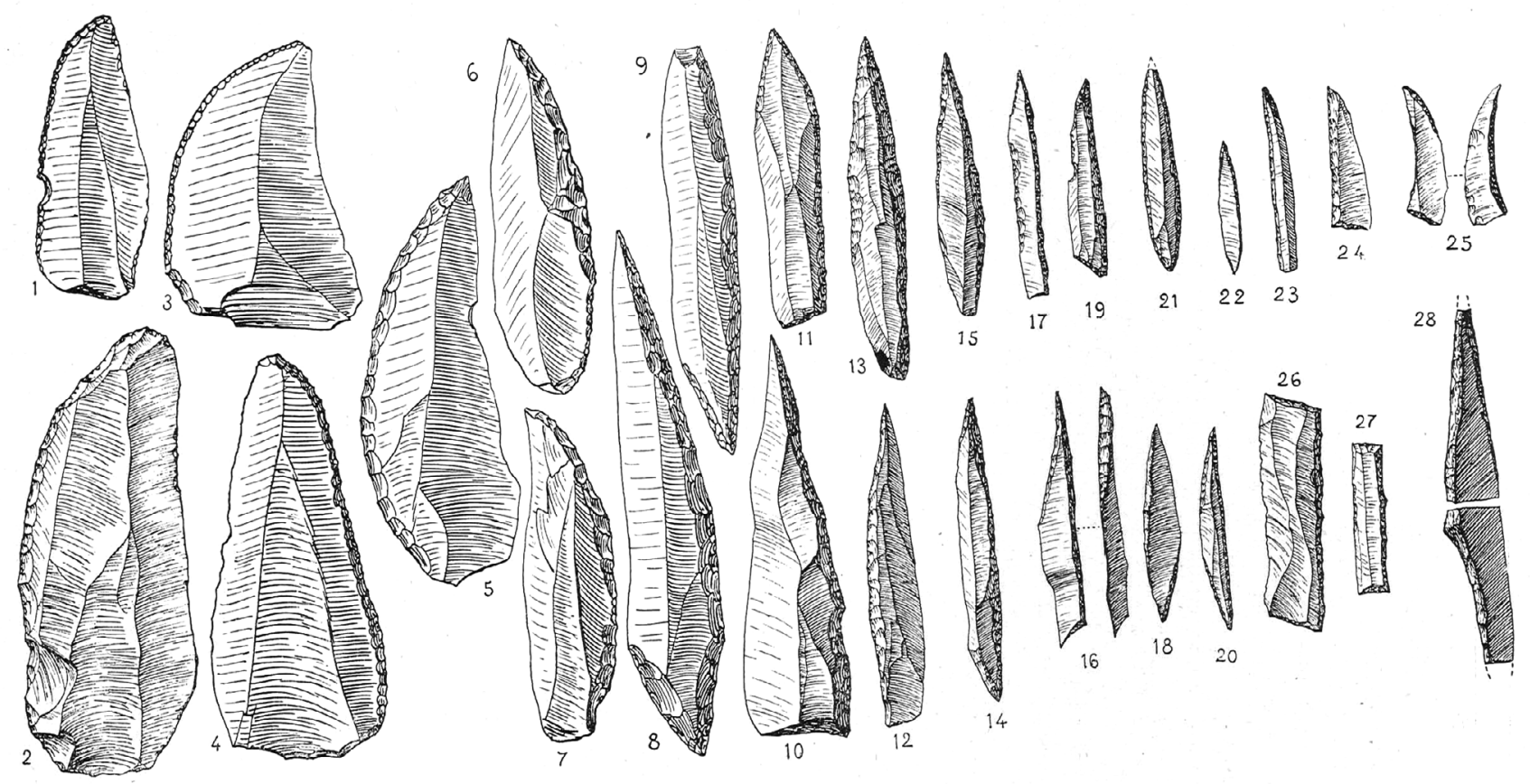

Figure 1 - «Évolution de la pointe aurignacienne à un tranchant rabattu à travers tout l'Aurignacien » (Breuil 1913).

Figure 1 - "Evolution of the Aurignacian point with a backed edge through all the Aurignacian" (Breuil 1913).

Cette césure contredit la vision dans laquelle D. Peyrony se situait quelques années plus tôt. Cette découverte s'articule dans un débat plus large qui tente de concilier l'idée d'une continuité dans l'évolution et l'observation de discontinuités dans la succession des industries (Guillomet-Malmassari 2012).

Dix ans plus tard, en 1932, le premier jalon du rapprochement entre les Aurignaciens inférieur et supérieur est mentionné :

« la culture de l'Aurignacien inférieur (type de Chatelperron) ne paraît pas avoir disparu complètement. Sur quelques points encore mal définis, elle semble s'être orientée vers celle de l'Aurignacien supérieur (type de La Gravette) " (Peyrony 1932).

La rupture reconnue à La Ferrassie est alors formalisée : " L'industrie qu'on est convenu d'appeler Aurignacien inférieur, mais qui, en fait n'a absolument rien de commun avec celle d'Aurignac, est caractérisée par les pointes à dos courbe et abattu dites type de Chatelperron " (Peyrony 1932). Cet aspect qu'il affirme en 1932 sera repris l'année suivante dans l'exposé complet de son modèle.

Onze ans après son premier doute sur la cohérence de l'Aurignacien, D. Peyrony définit le Périgordien (Peyrony 1933). Cette idée prend sa source principalement dans deux observations : la différence entre l'Aurignacien inférieur et l'Aurignacien moyen, la ressemblance entre
l'Aurignacien inférieur et l'Aurignacien supérieur. Elle se base principalement sur trois gisements : La Ferrassie, La Gravette et Laugerie-Haute. À l'aune de ces stratigraphies, il réinterprète les convergences techniques en termes de parentés (lignées), la localisation géographique des gisements en termes de territoires.

Les Aurignaciens inférieur et supérieur donnent naissance au Périgordien, l'Aurignacien moyen, devenant l'Aurignacien stricto sensu. Le Périgordien et l'Aurignacien sont alors conçus comme deux phyla évoluant en parallèle (fig. 2).

Ces lignées ne sauraient être envisagées seulement d'après la culture matérielle ; elles sont le fruit de deux races humaines différentes, celles de Combe-Capelle ${ }^{1}$ (dont le squelette fut découvert en 1909) et de Cromagnon. Cette association production/type humain est un élément structurant du modèle périgordien, dans la mesure où la culture ne peut être affranchie du prisme racial. Peyrony retrace ainsi le véritable combat qu'a mené l'homme de Combe-Capelle contre son prédécesseur, se retirant ensuite face à "l'arrivée des Cro-Magnon envahissant la région » (Peyrony 1933 - p. 556). Par la suite, ces tribus furent "anéanties ou refoulées par le retour d'une horde nombreuse de "Combe-Capelle 》" (Peyrony 1933 - p. 557).

(1) Ce schéma s'appuie sur les découvertes de sépultures moraves de Brno et Predmost, dont les défunts appartiendraient au groupe de Combe-Capelle, dont ils partageraient l'outillage. "Cette parenté des crânes de Combe-Capelle, de Brnô (Brun) et de Predmost vient renforcer l'hypothèse de l'évolution de la pointe de Châtelperron vers celles de La Gravette, de la pointe à cran atypique et de la pointe à soie » (Peyrony 1932). 
l'origine de cette couche, il imagine une "crue formidable, dont le courant venait buter, à quelque 10 kilomètres en aval, la falaise de Laugerie-Haute, (et qui) a certainement raviné l'abri et enlevé complètement jusqu'au sol naturel, tous les dépôts qui pouvaient y exister. C'est vraisemblablement à ce moment-là qu'on doit placer l'interstade würmien » (Peyrony et Peyrony 1938 - p 11).

La base des dépôts de Laugerie-Haute est ainsi rapportée au début du Paléolithique supérieur. Comme le remarque Peyrony (et d'autres avant lui), la stratigraphie de nombreux abris du Périgord débute par une couche d'éboulis calcaires, en mentionnant l'abri du Poisson, l'abri Castanet et La Gravette. II en déduit qu'à Laugerie-Haute : « le dépôt de base serait contemporain par partie des Périgordiens I et II et de l'Aurignacien I 》 (Peyrony et Peyrony 1938 p. 12).

En revanche, cette contemporanéité établie sur des bases sédimentaires, se trouve en contradiction avec les données climatiques. D. Peyrony constate que "la faune $d u$ Moustiérien final et du Périgordien I (niveau Chatelperron), quoique froide, n'est pas glaciale, tandis qu'il n'en est pas de même de celle de l'Aurignacien I (niveau des pointes à base fendue), où figurent le phoque du Groenland, le Lemming, le Renard polaire (abri Castanet), le Bœuf musqué (abri du Poisson), I'Isatis (abri Lartet), le Spermophile (Cro-Magnon et la Ferrassie) 》 (Peyrony et Peyrony 1938 - p. 11).

La position chronologique du Périgordien III est donc définie à partir d'un hiatus sédimentaire expliqué à partir de la séquence du Moustier, d'une comparaison sur une base sédimentaire $^{2}$ avec les abris régionaux et en dépit des divergences climatiques reconnues entre les horizons mis en parallèles. Cette dernière incohérence n'est pas retenue, car l'argument est, pour lui, suffisant pour démontrer ce qu'il pressent à partir du mobilier lithique :

" L'industrie de Laugerie-Haute s'apparente aux précédentes par ses lames à bord arqué partiellement abattu ou tronquées obliquement ; mais, par ses lames à bord abattu, à gibbosité, ses grattoirs larges ou discoïdes, elle tend vers la culture de La Gravette. Cette industrie paraît donc représenter l'étape intermédiaire entre les Périgordiens II et IV » (Peyrony et Peyrony 1938 - p. 20).

En résumé, D. Peyrony a conçu la chronologie périgordienne sur :

- Périgordien I et II : la succession stratigraphique de La Ferrassie ;

- Périgordien II et III : une corrélation chrono-sédimentaire entre Laugerie et le Moustier ;

- Périgordien III et IV : le caractère supposé intermédiaire de l'industrie de Laugerie avec les industries de type Châtelperron et de La Gravette ;

- Périgordien IV et V : l'aspect solutréen, donc plus récent, de la retouche de certaines pointes de La Font-Robert, dans la ligne de la première attribution de ce faciès par J. Bouyssonie.

\section{Conclusion de la première sériation}

Cette évolution de la pensée de D. Peyrony retrace dans ses grandes tendances, la manière dont il se démarque de la chronologie breuillienne. $\mathrm{H}$. Breuil émettra quelques réserves au sujet du Périgordien, "cette idée est très soutenable, bien qu'on puisse considérer comme non prouvé le parallélisme des deux développements et leur rattachement à des races définies. "D'ajouter, plus incisif : « II n'y a aucune raison stratigraphique d'accepter ce parallélisme » (Breuil 1937), remarques auxquelles D. Peyrony répondra en 1946, dans la seconde version du modèle.

Comme le souligne $\mathrm{H}$. Breuil, « il n'y a jusqu'ici nulle part interstratification des deux groupes industriels, Périgordien II-V et Aurignacien I-V, et partout, jusqu'ici, ceux-ci supportent partie de ceux-là, quand il y a contact » (Breuil 1937). Nous pourrions répondre à la place de $\mathrm{D}$. Peyrony, pour qui l'Aurignacien $\mathrm{V}$ de Laugerie-Haute constitue un argument de contemporanéité, mais la question n'est pas là. II ne s'agit pas d'énumérer les biais pourtant nombreux, mais de comprendre qu'au-delà du cadre chronologique reconnu par $\mathrm{H}$. Breuil à l'issue de la bataille aurignacienne, un changement d'approche global conduisit Peyrony à accorder une plus forte valeur à la convergence technique qu'à la succession stratigraphique. C'est effectivement l'interprétation chronologique de la succession stratigraphique qui les distingue.

Un des points de départ de ce modèle se situe donc un peu à l'avant du Grand abri de La Ferrassie et découle d'une observation de terrain. L'interprétation résultante est à l'origine d'une notion toujours présente dans notre approche des groupes humains fossiles: l'idée d'une discontinuité structurelle entre industries, idée déjà envisagée par Breuil. Dans ce contexte, elle est située entre les niveaux que nous attribuerions aujourd'hui au Châtelperronien et à l'Aurignacien initial. Si D. Peyrony perçoit que ces différences renvoient à une césure historique, il situe ce changement au rang des nombreuses guerres tribales qui ont jalonné la préhistoire.

Le Périgordien peut ainsi être abordé comme la recherche de continuité, alors que les nouvelles fouilles mettent en évidence l'absence de linéarité dans l'évolution des industries. Le système périgordien conduit donc à réinterpréter les données géologiques pour chercher une continuité sur la base des industries dans une vision linéaire de l'évolution. Pour expliquer ces différences, le prisme racial est dominant, la technique étant synonyme de culturel et de biologique.

Dans la mise en place de ce schéma, de nombreux acteurs sont intervenus. Ses échanges avec certains préhistoriens, comme Carthaillac, dans le cadre de ses fouilles à Grimaldi, tiennent une place notable. L. Bardon, les frères $A$. et $J$. Bouyssonie figurent en bonne position dans l'élaboration de ce modèle. Pour s'en convaincre, reprenons la conclusion de J. Bouyssonie à l'étude de Bos-del-Ser : « Faut-il aller plus loin et rattacher les industries à des races humaines ? Les pièces à belles retouches type Aurignac, Gorge d'Enfer, Bouïtou, fabriquées par la race de Cro-Magnon; les pièces à bord rabattu, Abri Audi, Chatel-Perron, Bos-del-Ser,

(2) En 1939, Peyrony reprend cette observation et établit une sériation chronologique, à partir de comparaisons sédimentaires et climatiques, élargie au Paléolithique supérieur (Peyrony 1939). 
Grimaldi, puis Gravette et Font-Robert, œuvre de la race de Grimaldi. Les hommes de ces deux races se seraient disputé les abris de Laussel » (Bouyssonie 1923 - p. 622).

\section{2 - La seconde sériation}

Deux découvertes, à La Gravette et à Dufour, vont conduire D. Peyrony à aménager ce premier schéma.

F. Lacorre met au jour à La Gravette un niveau à fléchettes (Lacorre 1933a, 1933b) à la base de la séquence gravettienne (Périgordien IV) et au-dessus de l'Aurignacien. Ce dernier invite D. Peyrony à visiter ce gisement en 1935 et à s'intéresser à la position originale de ce niveau.

Dans sa compréhension de la chrono-stratigraphie, ce niveau à fléchettes par: "Sa position le fait contemporain du niveau des pointes en os losangiques aplaties de la Ferrassie (Aurign. II) et de celui de la base de LaugerieHaute (Périgordien III) » (Peyrony 1936 - p. 617). L’idée de lacunes stratigraphiques est absente de ce raisonnement, pourtant évoquée à d'autres moments. La succession stratigraphique désigne, dans ce cas, une succession chronologique directe.

Pour D. Peyrony, la reconnaissance du niveau à fléchettes, postérieur à l'Aurignacien I et sous-jacent au Périgordien IV, renforce cette idée d'une évolution de la lamelle de La Ferrassie et de Bos-del-Ser vers les petites pièces foliacées trouvées par F. Lacorre. En 1936, aucune subdivision supplémentaire du Périgordien n'est pourtant entamée, dans son premier réajustement du Périgordien (fig. 3). D'après son modèle d'évolution, les lamelles de
Bos-del-Ser et les fléchettes ne devraient pourtant pas se situer dans la lignée directe d'évolution morphologique des pointes à dos des Périgordiens I, III et IV, comme en témoigneront les discussions à venir autour du Périgordien II. Le niveau bayacien a également livré des spécimens de fléchettes qui possèdent un dos : les fléchettes à dos courbe (fig. 4) et les fléchettes asymétriques (Pesesse 2008). Ces pièces s'intègrent plus dans l'idée d'une évolution de la pointe à dos et auraient pu fournir un argument supplémentaire à $\mathrm{D}$. Peyrony, mais elles ne sont pas mentionnées spécifiquement (Peyrony 1936). C'est donc notamment par défaut de pièces similaires dans la séquence aurignacienne constituée en parallèle que tous ces supports légers retouchés s'intègrent dans le même phylum.

A. et J. Bouyssonie, du fait de leurs nombreuses fouilles, vont une nouvelle fois intervenir dans la sériation peyroniste, avec la publication de la Grotte Dufour, située à proximité de Bos-del-Ser et fouillée en 1938. J. Bouyssonie présente cette industrie caractérisée par une abondante série de "lamelles finement retouchées, par retouches alternes ", mais, dit-il, " nos troglodytes ne fabriquaient plus de couteaux à dos incurvé du type Chatelperron et pas encore de couteaux rectilignes du genre Gravette » (Bouyssonie 1944 - p. 192).

En 1942, J. Bouyssonie et D. Peyrony étudient ensemble cette collection. Ce dernier de conclure sur " cet heureux événement »: « je remarquai ce qu'avait fait déjà Bouyssonie, qu'il y avait là toutes les pièces de Bos-delSer, moins les formes moustériennes et les pointes et lames tronquées type Chatelperron. Le problème se trouvait ainsi résolu » (Peyrony 1946 - p. 232).

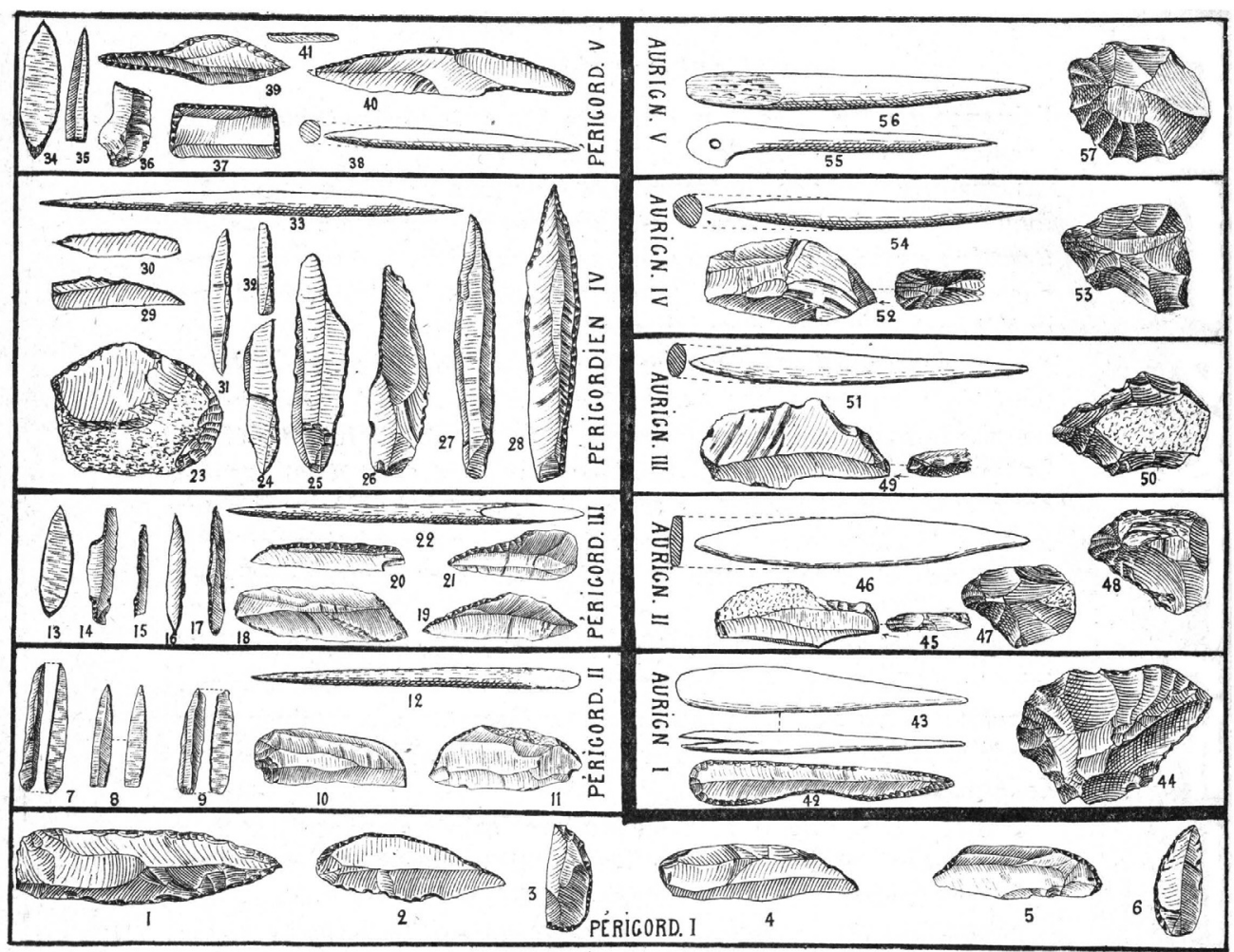

Figure 3 - Le Périgordien en 1936 (Peyrony 1936).

Figure 3 - The Perigordian in 1936 (Peyrony 1936). 

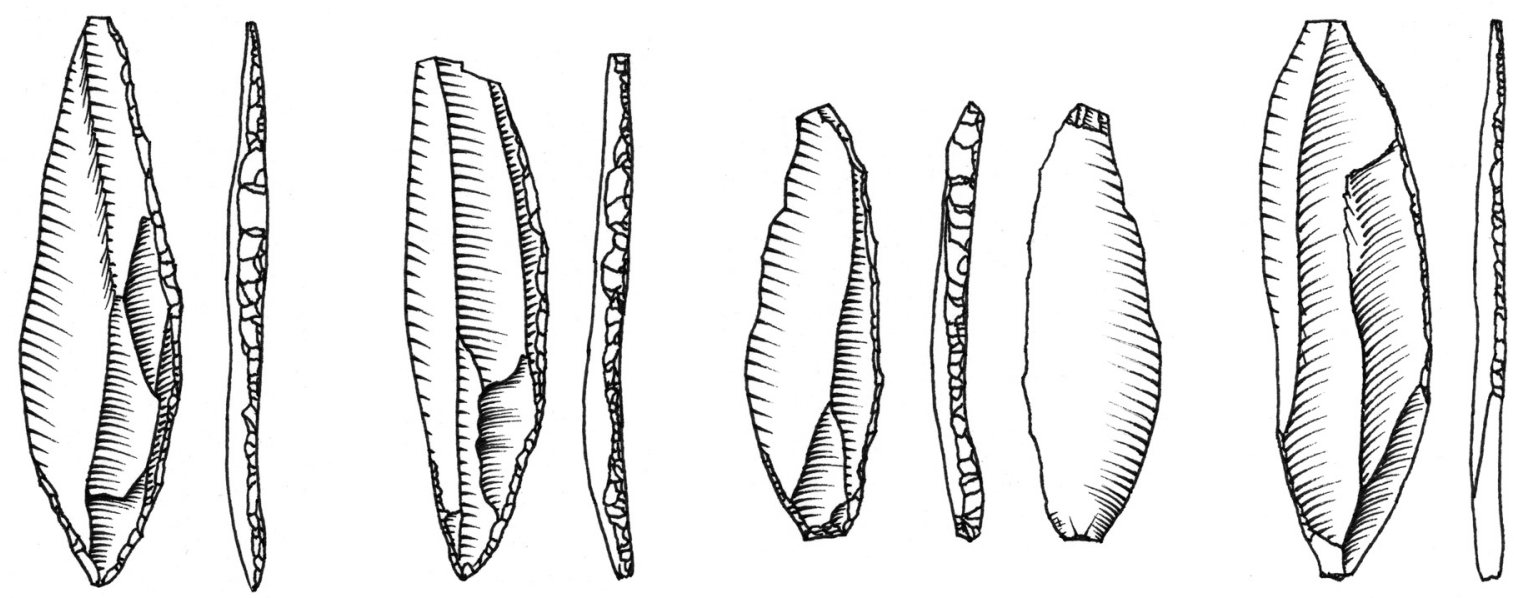

Figure 4 - La Gravette, niveau bayacien, fléchettes à dos courbe, éch. 1/1 (Pesesse 2008).

Figure 4 - La Gravette, Bayacian level, flechette with curved back, scale: 1/1 (Pesesse 2008).

Cette découverte répond directement à l'observation réalisée par D. Peyrony à La Ferrassie en 1920 : les lamelles à retouches alternes et les pointes de Châtelperron ne sont pas toujours associées. II interprète alors Bos-del-Ser - et Pair-non-Pair - comme résultant de mélanges (Peyrony 1946).

Pour réaliser ces comparaisons, de Pair-non-Pair D. Peyrony retient les «fines lamelles à retouches marginales » (Peyrony 1946). Distingue-t-il dans cet ensemble de lamelles, celles à retouche directe (Cheynier et Daleau, 1963, fig. 23 et 27) et celles à retouche inverse (fig. 5) ? Avant Dufour, des lamelles à retouches inverses ont donc été retrouvées à Pair-non-Pair, Grimaldi, La Ferrassie ou à Bos-del-Ser où elles furent rencontrées en nombre. La publication de Dufour est pourtant postérieure de vingt ans à celle de Bos-del-Ser. Dans ce site identifié par L. Bardon deux décennies avant sa publication, le moment précis de leur découverte nous échappe. D’après les subventions de fouilles demandées à l'IPH en 1912 et à l'AFAS en 1921, nous pouvons nous demander si J. Bouyssonie les avait trouvées à Bos-del-Ser avant la publication de D. Peyrony. Ce dernier ne les mentionne pas en 1922 mais en avait-il connaissance ? Elles seront dénommées "lamelles de Dufour » ou "lamelles du type Dufour » (Pradel 1952a), puis «lamelles Dufour » (Sonneville-Bordes 1955a).

Alors que ses fouilles de La Ferrassie l'avaient amené au même constat, D. Peyrony n'avait pas poursuivi son raisonnement. Tout en énonçant que le Périgordien II constitue la suite du Périgordien I, il remarquait que les lames du Périgordien II ne présentaient aucun caractère des pointes du Périgordien I. C'est cette contradiction qu'il va tenter de résoudre dans la seconde version du modèle. Ce problème de continuité recoupe celui d'une autre différence entre Périgordien et Aurignacien, l'homogénéité de ces deux entités. Cet aspect introduit sa révision :

" L'ensemble des faciès aurignaciens est assez homogène ; on retrouve dans chacun d'eux les caractères généraux de toute la culture. II n'en est pas de même de ceux que j'ai classés dans le Périgordien » (Peyrony 1946 p. 232).
Alors que la mixité typologique de Bos-del-Ser était considérée comme un caractère transitionnel, la découverte de Dufour réfute cette possibilité d'un continuum direct entre Périgordien I et Périgordien II. Déjà partie prenante de la première version du Périgordien, la valeur territoriale est ici amplifiée pour permettre à ces groupes d'exister : “De ces observations et d'autres, il ressort qu'en Dordogne, Corrèze et Gironde, on remarque dans le Périgordien, deux groupes distincts présentant chacun des faciès évolutifs : " (Peyrony 1946 - p. 232). II définit alors plusieurs groupes:

- Dordogne : Périgordien I, type La Ferrassie, puis La Gravette, niveau à fléchettes, puis La Forêt à Tursac (il s'agit vraisemblablement de l'abri appelé aujourd'hui du Facteur)

À ce stade, le niveau à fléchettes ne peut toujours pas être vraiment intégré. II devrait l'être au Périgordien II, mais ce niveau se situe en Dordogne. D. Peyrony ne le mentionne donc que secondairement. II le situe entre le niveau à pointes de Châtelperron et les niveaux classiques de La Gravette, sans préciser sa position par rapport au Périgordien III et en oubliant ses affinités avec le Périgordien II.

- Corrèze : Périgordien II, type Dufour, puis Font-Yves, puis Noailles,

L'apparition de la grotte de Noailles, dans ce second volet, semble plus s'expliquer comme la recherche d'un jalon de la fin du Périgordien en Corrèze. Son industrie offre peu de points de comparaisons avec le Périgordien II. Contrairement aux nouveaux gisements qu'il intègre, la Grotte Chez Serre, à Noailles, n'a d'ailleurs fait l'objet d'aucune nouvelle publication depuis la première version, dont elle est absente.

- Gironde : seul le premier faciès (type Dufour) a été découvert à Pair-non-Pair. 

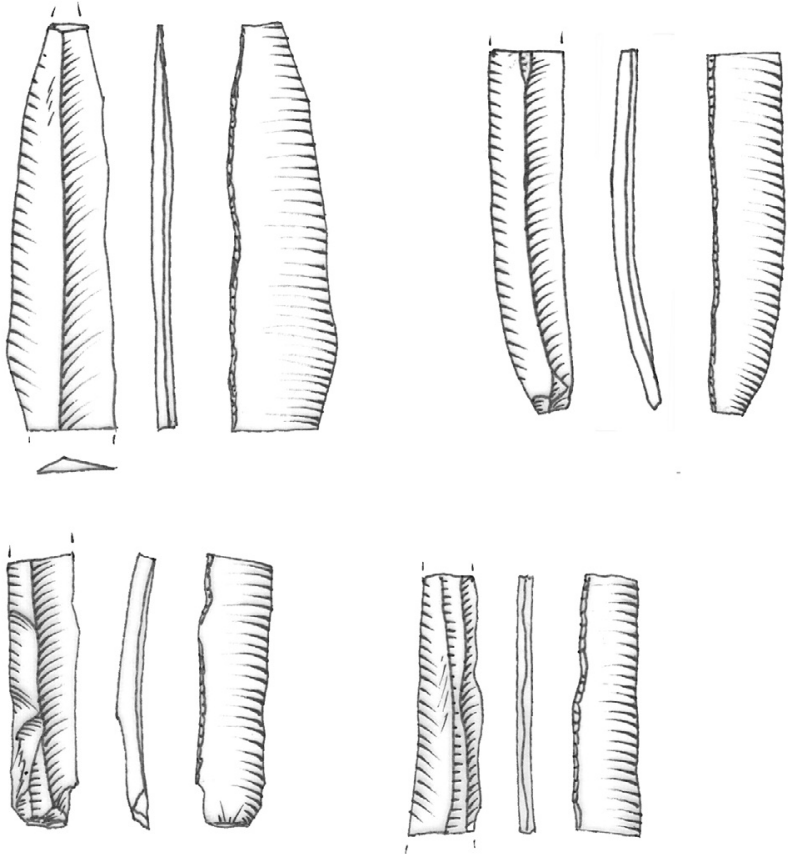

Comme il le mentionne plus haut dans l'article, D. Peyrony sait pourtant bien que ce gisement a livré une belle série de pointes de Châtelperron.

\section{Conclusion de la seconde sériation}

Pour D. Peyrony, cette seconde version est donc plus logique. Malgré tout, il utilise de manière partielle les données pour les incorporer dans son système, notamment en ce qui concerne Pair-non-Pair, Noailles et le niveau à fléchettes de La Gravette.

En dépit de ses efforts destinés à mettre en phase les données archéologiques avec son propre système, et non l'inverse, ce deuxième stade intègre de nouveaux paramètres. Les Périgordiens I et II, perdent leur caractère essentiellement chronologique pour intégrer une chronologie interne à valeur régionale, plutôt même départementale. Ils deviennent ainsi des Périgordiens au sein du Périgordien. Ils révèlent en effet le même processus de reconstruction que le premier Périgordien visà-vis de l'Aurignacien. Aux mêmes données (impossibilité de concevoir l'évolution d'une industrie à l'autre, remise en question des associations stratigraphiques à partir d'une nouvelle fouille, perspective régionaliste, convergences techniques), D. Peyrony propose un nouveau soussystème répondant aux normes du précédent : un sousPérigordien qui comme le Périgordien et l'Aurignacien voit l'évolution parallèle de deux groupes sur des territoires légèrement différents.

Cette complexification du premier système repose donc sur les mêmes fondements interprétatifs que le précédent. Les sous-groupes du Périgordien et de l'Aurignacien sont toujours les «tribus de civilisations différentes » (Peyrony 1946). C'est en revanche à une échelle géographique bien plus vaste que D. Peyrony dresse son schéma. La
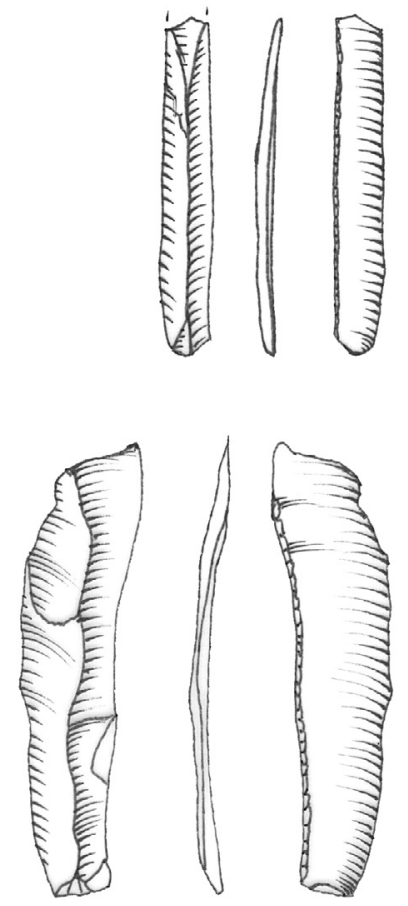

Figure 5 - Lamelles à retouches sur face plane de Pair-non-Pair, niveau $K, K D$ et 4 , éch. 1/1.

Figure 5 - Retouch on flat face bladelet from Pair-nonPair, level K, KD and 4, scale: $1 / 1$. recherche d'ensembles du Périgordien « pur » a en effet porté son attention vers le Proche Orient à la suite des travaux de D. Garrod, cherchant des relais par "Surène » (Siuren) en Crimée, puis Krems en Autriche. Ce changement d'échelle est un aspect notable de l'évolution de la pensée de D. Peyrony, présent dès 1932. Au fil des publications, l'espace géographique pris en compte est en effet croissant (Peyrony 1948).

Le Périgordien résulte de l'impossibilité de concevoir, autrement que dans une logique de continuité, les discordances révélées par les données archéologiques. L'amélioration des observations de terrain, la prise en compte d'un nombre croissant d'aspects, sont toujours envisagées dans une obligation de continuum, fut-ce par le développement parallèle de deux lignées.

Le Périgordien fait appel à « des données morphologiques, stratigraphiques, paléontologiques et géologiques qui peuvent être vérifiées 》 (Peyrony 1940 - p. 708), appliquées avec des notions héritées du $\mathrm{XIX}^{\circ}$ siècle et non questionnées. Les référents de $\mathrm{D}$. Peyrony sont parfois presque bibliques, lorsqu'il fait du Périgord, un lieu « de pèlerinage au pays des grottes et des abris, dont la tradition devait en faire le «paradis terrestre » des tribus errantes de cette époque " (Peyrony 1940 - p. 704). Même dans la seconde version du Périgordien, publiée en 1946, les conceptions sous-jacentes semblent avoir peu évolué.

\section{3 - Heures et malheurs du Périgordien}

En dépit des biais évoqués précédemment, le Périgordien rencontra un franc succès chez les Préhistoriens. L'apport de nouvelles fouilles vint toutefois modifier certains points de la sériation proposée. Après la mort du « père du Périgordien », une certaine division s'instaure au sein de la 
communauté scientifique. Entre 1954 et 1960, une profusion d'articles va aborder le Périgordien, questionnant parfois même ses fondements.

En France, H. Delporte produit la première critique du Périgordien. Cette critique ne concerne pas l'attribution d'un site ni la caractérisation d'un faciès mais la vision globale de D. Peyrony. D'abord il constate, à partir du matériel archéologique, la plus grande parenté des industries du type Châtelperron avec le Moustérien et celles du type La Gravette avec le pré-solutréen et le solutréen. H. Delporte de proposer :

"N'y aurait-il donc pas intérêt à abandonner le terme de Périgordien, d'ailleurs non conforme aux règles de la terminologie, et à distinguer deux cycles de civilisation différents : le Castelperronien et le Gravettien ? » (Delporte 1954 - p. 46, en s'appuyant sur D. Garrod).

Cette proposition s'accompagne d'une remise en question complète des modèles interprétatifs :

- " il apparaît de plus en plus que la thèse de l'évolution linéaire en matière humaine ne peut être qu'un leurre. L'hypothèse de l'évolution buissonnante semble beaucoup plus juste » (Delporte 1954 - p. 46),

- « il est donc probable que la question de la liaison entre race humaine et civilisation, tout au moins pour le Périgordien, est beaucoup plus complexe qu'on peut le penser » (Delporte 1954 - p. 47).

H. Delporte formule ensuite sa propre conception de la recherche préhistorique :

"Ce ne sera plus la voie de cheminement d'un groupe et d'une civilisation homogène que nous devrons essayer de déterminer, mais celle des courants de civilisation; et cela en tenant compte des conditions géographiques et climatologiques, mais aussi humaines " (Delporte 1954 p. 48).

Ce court article s'inscrit donc, d'un point de vue méthodologique et conceptuel, en faux par rapport à la pensée peyroniste. Delporte remet en cause les fondements même du modèle Périgordien. Cette publication pourrait être considérée comme un tournant théorique, presque un manifeste. L'idée du buissonnement qui s'oppose à la linéarité sera d'ailleurs largement reprise par la suite (Sonneville-Bordes 1955a).

Mais cette remise en question ne fera pas école. S'en suivra un véritable " conflit ouvert au sein de la SPF » (Lacorre 1956 - p. 140) qui aboutira à un clivage de la communauté dont les positions seront essentiellement imperméables ${ }^{3}$. La réponse faite aux observations de $\mathrm{H}$. Delporte sera particulièrement vive (Sonneville-Bordes 1955b ; Combier 1955 ; Pradel 1955). La réponse à la contradiction d'un constat de polymorphie (Sonneville-Bordes 1955a) et à un concept de linéarité (Pradel 1955) n'est pas apportée. Le
Périgordien survit à cette première attaque et trouve des défenseurs attitrés. L. Pradel remet d'ailleurs le concept de buissonnement au compte de D. Peyrony, en le rapprochant de la notion d' « interférences » qu'il rapporte à la publication de 1946 de cet auteur (Pradel 1955), bien que ce terme n'y figure pas (Peyrony 1946). Ce n'est donc pas sur les fondements des interprétations de D. Peyrony, mais sur le Périgordien $\mathrm{II}^{4}$, que porteront les critiques.

L'occasion est donnée par la fouille de Caminade et la découverte de lamelles à retouches alternes en contexte aurignacien. À partir de Bos-del-Ser, Mochi, Pair-non-Pair, D. de Sonneville-Bordes suppose tout d'abord que «la lamelle est le fossile directeur de l'Aurignacien ancien, stratigraphiquement situé entre le Périgordien I et l'Aurignacien typique I, autrement dit que l'ancien Périgordien II est en réalité un Aurignacien ancien qui s'empare de sa position stratigraphique et de son fossiledirecteur » (Sonneville-Bordes 1955a - p. 200). Mais leur présence à Caminade n'abonde pas dans cette acceptation chronologique. L'Aurignacien est alors envisagé « en deux branches, l'une avec, l'autre sans lamelles » (SonnevilleBordes 1955a - p. 200). "La « lamelle Dufour » étant ici interprétée comme un instrument aurignacien, et non plus comme un fossile directeur du Périgordien II, le Périgordien II, tel que l'avait défini $D$. Peyrony, disparaît de la nomenclature » (Sonneville-Bordes 1955c - p. 359).

Ce point est intéressant car D. de Sonneville-Bordes tout en reconnaissant les incohérences du Périgordien $\|^{5}$, reproduit le mécanisme. II existe une convergence technique, les lamelles, celles-ci n'ont pas de valeur chrono-stratigraphique ${ }^{6}$, elles signent alors peut-être deux lignées au sein de l'Aurignacien. Le modèle périgordien est donc reproduit, mais appliqué en contexte aurignacien. Le schéma de D. Peyrony est ainsi corrigé, affiné, mais absolument pas modifié. L'attaque du Périgordien II renforce le Périgordien.

Si les fondements n'ont pas varié jusque là, un élément allait tout de même le modifier. D. Peyrony ne publia pas le tableau synoptique de son second système, mais remit un tableau manuscrit à $F$. Lacorre, qui le diffusa à d'autres préhistoriens (Pradel 1952a qui intégra des additions personnelles) avant d'en publier lui-même une version (Lacorre 1956). Dans ce tableau (fig. 6), tout en figurant en parallèle les deux lignées périgordiennes et aurignacienne, un décalage chronologique s'opère entre les groupes du Périgordien, s'ajoutant au décalage géographique. Ceci diffère donc de la vision initiale de D. Peyrony sur l'évolution globalement synchrone des groupes, fondée sur les stratigraphies du Moustier et de Laugerie. Un écart chronologique s'insère alors entre Périgordiens I et II et Périgordiens III, IV, V.

Suite aux travaux qu'il conduit à la Grotte des Enfants avec L. Barral, et aux études de D. de Sonneville-Bordes,

(3) Ce n'est peut-être pas le cas pour F. Lacorre et A. Cheynier.

(4) Le Périgordien II : " créé tardivement, faute de mieux, doit être reconsidéré à la lumière de découvertes récentes " (Pradel 1955, p. 607), dont « la personnalité ne nous semble pas clairement définie, ni même l'existence exactement démontrée " (Sonneville-Bordes 1955a, p. 187).

(5) Incohérences largement liées aux gisements attribués postérieurement à D. Peyrony au Périgordien II.

(6) D. de Sonneville-Bordes évoque toutefois les biais éventuels liés à l'absence de tamisage. 


\section{PERIGORDIEN}

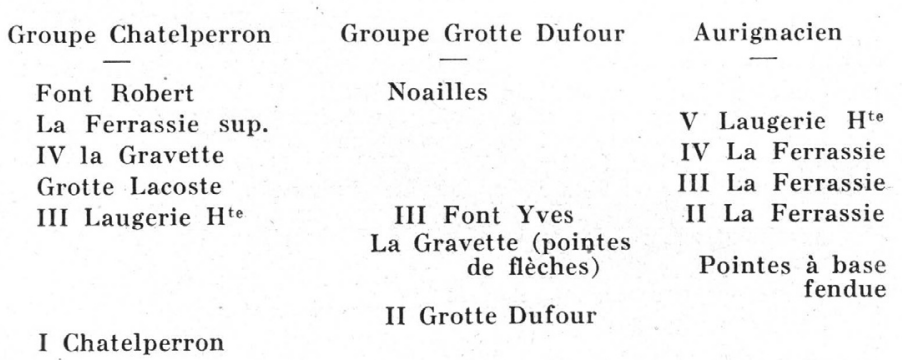

Figure 6 - Copie du tableau manuscrit de 1946 de Denis Peyrony (Lacorre 1956).

Figure 6 - Copy of the handwritten chart of 1946 from Denis Peyrony (Lacorre 1956).
F. Lacorre constate que les outillages à lamelles retouchées se rapportent en partie à des industries aurignaciennes, tout en conservant leur spécificité qui ne permet pas de faire disparaître le Périgordien II. L'option choisie est donc la création du groupe «Corrézien », option mixte affirmant la spécificité de ces industries et ménageant les susceptibilités des tenants du Périgordien (Lacorre 1956). Cette proposition fut suivie par L. Pradel, notamment en raison de la différence de caractère entre la retouche des industries corréziennes et périgordiennes (Pradel 1956). Delporte fera preuve une nouvelle fois de rigueur terminologique, en remarquant qu'il y a du périgordien en Corrèze, que la Corrèze ne correspond pas à une entité, et qu'il faudrait plutôt prendre en compte le contexte géologique correspondant pour créer le «Brivien 》 (Delporte 1957a). Et pourquoi pas le « Dufourtien » (Pradel 1952a) ?

À cette ramification progressive du Périgordien, A. Cheynier va opposer l'argument stratigraphique (Cheynier 1955) et s'appuyer sur les 6000 ans de l'Aurignacien. Toutefois, le déclic viendra de ses fouilles au Cirque de la Patrie. La morphologie des pointes de La Gravette exhumées ne s'intègre pas dans une évolution directe depuis les pointes de Châtelperron. Ceci va modifier ses conceptions initiales ${ }^{7}$ (Cheynier 1953) et l'amener à reconsidérer le séquençage du Périgordien. Le Castelperronien remplace alors le Périgordien I et le Périgordien désigne les industries situées entre l'Aurignacien et le Solutréen. D'obédience breuillienne, A Cheynier pallie l'absence de lien entre Aurignacien et Périgordien en observant que "les Périgordiens ont substitué les Pointes en pierre aux Pointes en os » des Aurignaciens (Cheynier 1955 - p. 517). La continuité de l'évolution est ainsi compatible avec la discontinuité de la succession des industries, grâce à cette possibilité d'un transfert technique de la pierre à l'os. L'idée d'H. Breuil subsiste. II reprend également le terme de Gravétien (Cheynier 1959).

La valeur chronologique relative de la succession stratigraphique, dans la lignée de $\mathrm{H}$. Breuil, refait surface chez F. Lacorre. II reprend le modèle de D. Peyrony et y intègre ses propres observations, dans lesquelles la Grotte des Enfants tient une place importante. II aboutit à de nouvelles subdivisions, complexifiant encore le tableau évolutif. Mais F. Lacorre se différencie de D. Peyrony par son approche de la stratigraphie. II constate en effet que le « Gravétien » est toujours sus-jacent à l'Aurignacien, que l'Aurignacien ne surmonte que le Châtelperronien, à l'exception de trois sites présentant un « mélange » de Périgordien et d'Aurignacien. Cela s'intègre tout de même au sein d'un phylum Périgordien (Lacorre 1959a et b).

L'idée du Gravettien émerge donc de nouveau entre 1953 et 1960. Le terme "Gravétien » se répand dans la littérature, sa position chrono-stratigraphique réenvisagée et ses liens morpho-phylétiques avec le «Chatelpéronien » reconsidérés. Dans ce contexte favorable, A. Cheynier propose le « Gravétien » en 1960 (Cheynier 1960), année de la publication du gisement éponyme (Lacorre 1960). Tout semble enfin prêt pour accueillir le « Gravétien ».

\section{4 - L’apparition du Gravétien ?}

Cette effervescence aurait pu engendrer la naissance du Gravétien, mais comme rien n'est linéaire :

"Nous avions, en effet, admis, à la suite de plusieurs auteurs, la division du Périgordien de $D$. Peyrony en deux industries différentes, pour lesquelles nous avions accepté ou proposé les termes de CASTELPERRONIEN et de GRAVETTIEN (...) on peut admettre, avec Mme Bordes, que le terme de Gravettien ne se justifie pas. C'est pourquol nous utiliserons pour le complexe aurignaco-périgordien, l'échelle stratigraphique d'ensemble suivante: III. PERIGORDIEN = Périgordien supérieur de Peyrony ; II. AURIGNACIEN = Aurignacien sens stricto de Peyrony ; I. CASTELPERRONIEN = Périgordien inférieur de Peyrony »(Delporte 1957b).

H. Delporte invoque :

- l'absence de lame de La Gravette, dans le niveau à burins de Noailles du Facteur ${ }^{8}$ qu'il fouille depuis 1955,

- l'absence de publication du gisement éponyme,

- la nouvelle datation sédimentaire de Lommel (Limbourg belge) à l'Alleröd, dont l'industrie est rapportée à un périgordien à burins de Noailles, ce qui révèle « l'influence périgordienne au sein des complexes mésolithiques » belges.

(7) En 1953, il réaffirmait pourtant l'évolution de la lame à bord abattu : Audi-Châtelperron-Laugerie-Haute-Cirque de la Patrie - La Gravette (Cheynier 1953).

(8) Ces pièces sont pourtant nombreuses dans la collection E. Peyrony que H. Delporte a étudiée. 
En revenant sur l'idée gravettienne, $\mathrm{H}$. Delporte poursuit tout de même la division du Périgordien en Périgordiens inférieur et supérieur, écart suggéré dans le tableau manuscrit de D. Peyrony.

Les fouilles de H. L. Movius à l'abri Pataud en 1958 et de F. Bordes à Laugerie-Haute (Bordes 1958) vont accroître le fossé séparant les deux horizons du Périgordien, en remettant en cause la position chrono-stratigraphique du Périgordien III, qui devient le Périgordien VI.

Mais aucun nouveau jalon, ni explication ne pallie la fin du Périgordien III : “ Ce périgordien dit moyen, par D. Peyrony, dont l'étude détaillée de Laugerie-Haute peut éclaircir les relations d'une part avec le Périgordien ancien, d'autre part avec le Périgordien supérieur, se révèlera peut-être comme une branche d'évolution parallèle, partiellement du moins, avec le Périgordien supérieur des sites classiques" (Sonneville-Bordes 1960 - p. 217). C'est sur la suggestion d'une hypothétique nouvelle lignée Périgordienne que se termine la polémique du Périgordien III, dernier « chaînon » intermédiaire de la lignée périgordienne.

Dès lors, la contemporanéité de ces phyla ne repose plus que sur l'interstratification de l'Aurignacien $V$ et du Protomagdalénien, qui devra être réinterprété comme du Périgordien final (Bordes et Sonneville-Bordes 1966) pour argumenter de cette contemporanéité.

D. de Sonneville-Bordes reconnaît ponctuellement le manque de fondements de ces regroupements, sans en tirer les conséquences logiques : “Quoi qu'il en soit, l'hétérogénéité de l'Aurignacien et du Périgordien moyen et supérieur dans les divers aspects que nous en avons étudiés est de toute évidence absolue » (Sonneville-Bordes 1960 - p. 218).

Le Périgordien ne subsiste donc que grâce à un réel effort pour définir des jalons intermédiaires. Cette nécessité prend une nouvelle fois le pas sur les observations archéologiques. Quelques gisements deviennent ainsi les garants de ce passage. La polémique entourant l'attribution chronologique des gisements à pointes à dos abrupt des gisements de la Vienne, le Fontenioux, puis les Cottés (Daniel 1952 ; Pradel 1952b ; Sonneville-Bordes 1952 ; Delporte 1953 ; Pradel 1953 ; Sonneville-Bordes 1960 ; Pradel 1961 ; Laplace 1966) prend ainsi tout sons sens.

Seul un argument décisif serait à même de restaurer l'unité du Périgordien, remise en cause par ses détracteurs comme par certains de ses défenseurs. Le salut viendra des marges du Lot et de la Dordogne à la suite des fouilles de F. Bordes et J. Labrot au Roc-de-Combe et de F. Champagne et R. Espitalié au Piage. L'absence de jalons clairs entre Périgordiens inférieur et supérieur sera supplantée par la reconnaissance des inter-stratifications Périgordien/Aurignacien dans ces gisements voisins (Bordes et Labrot 1967 ; Champagne et Espitalié 1967) : "La théorie de D. Peyrony sort donc renforcée de ces dernières découvertes » (Bordes et Labrot 1967 - p. 28).

Ces nouveaux résultats tombent à point nommé, après la disparition des Périgordiens II et III. F. Bordes reproduit l'idée du caractère absolu de la datation par comparaisons stratigraphiques, en corrélant les séquences de La Ferrassie et du Roc de Combe-Capelle, il réaffirme la contemporanéité du Périgordien IV et des Aurignaciens III-
IV, tout en la nuançant des précautions scientifiques « modernes »: analyse pollinique, sédimentologique, datations. Cet « arsenal scientifique » ne sert d'ailleurs qu'à valider l'hypothèse défendue a priori. Le Périgordien IV devient le Périgordien moyen, remplaçant ainsi le défunt Périgordien III, jalon nécessaire pour maintenir ce modèle. L'idée breuillienne d'une évolution de la Châtelperron à La Gravette va servir une nouvelle fois d'argument absolu. Comment concevoir que deux outils si proches, (dont les intermédiaires seraient à chercher dans la Vienne), ne procèdent pas d'une lignée identique ? Cette possibilité n'est toujours pas acceptée.

La position géographique des sites lotois en marge de la région classique, comme la Corrèze et la Vienne, va une fois de plus servir d'argument, zone périphérique, zone de refuge où la succession des industries diffère quelque peu. Hormis le caractère anthropologique des distinctions phylétiques, le raisonnement de $\mathrm{F}$. Bordes se superpose clairement à celui de D. Peyrony. Le Périgordien est réaffirmé.

La publication tant attendue du gisement de La Gravette n'abondera pas dans le sens du Gravettien. Les couches rouge et noire ont livré un important outillage à dos (Lacorre 1960 ; Pesesse 2013). Cette technique concerne de nombreuses catégories d'outils. Pour F. Lacorre, cet outillage renforce les liens avec les ensembles à pointes de Châtelperron (fig. 7).

\section{5 - L'extinction des lignées}

L'appellation périgordien, abandonnée dans certains pays limitrophes (en suivant G. Laplace notamment), perdure en France. L'apparition de la datation absolue ne parvient pas à consommer le hiatus chronologique entre Périgordien inférieur et supérieur. Aucune avancée archéologique, méthodologique ni conceptuelle ne vient effriter ce modèle.

Curieusement, la voix de $\mathrm{H}$. Delporte posera à nouveau la question de l'unité périgordienne. Alors que ses fouilles à l'Abri du Facteur et à la Rochette, l'avaient guidé dans le sillage du Périgordien, la reprise de terrain de La Ferrassie allait influencer d'une toute autre manière sa perception de l'évolution des groupes humains. La stratigraphie du Grand Abri est à l'origine de ce nouveau changement d'opinion, soutenu par la généralisation des datations:

" les travaux récents ont montré qu'il existe un Périgordien ancien (Périgordien I), qui se situe aux environs de 35000 av. J.C. et un Périgordien récent (Périgordiens IV, V et VI), qui peut être daté des environs de 23000 à $20000 \mathrm{av}$. JC.. A notre connaissance, il n'existe pas d'industries « de transition " entre le Périgordien ancien et le Périgordien récent ; pendant toute la séquence aurignacienne, représentée par les couches $F, H, H^{\prime}$ et $H$ "' de la Ferrassie, couches qui correspondent à un remplissage de près de trois mètres d'épaisseur, la technique du bord abattu à totalement disparu, et nous ne comprenons pas comment il serait possible d'établir une filiation, quelle qu'elle soit, entre deux ensembles culturels séparés l'un de l'autre par plus de 10000 ans. Pour le moment, les fouilles de la Ferrassie n'ont livré aucun élément qui puisse contribuer à la solution de ce problème » (Delporte 1969 - p. 22). Déclencheur initial du Périgordien, La Ferrassie se trouve un demi-siècle plus tard, à l'origine du Gravettien. 
LA GRAVETTE

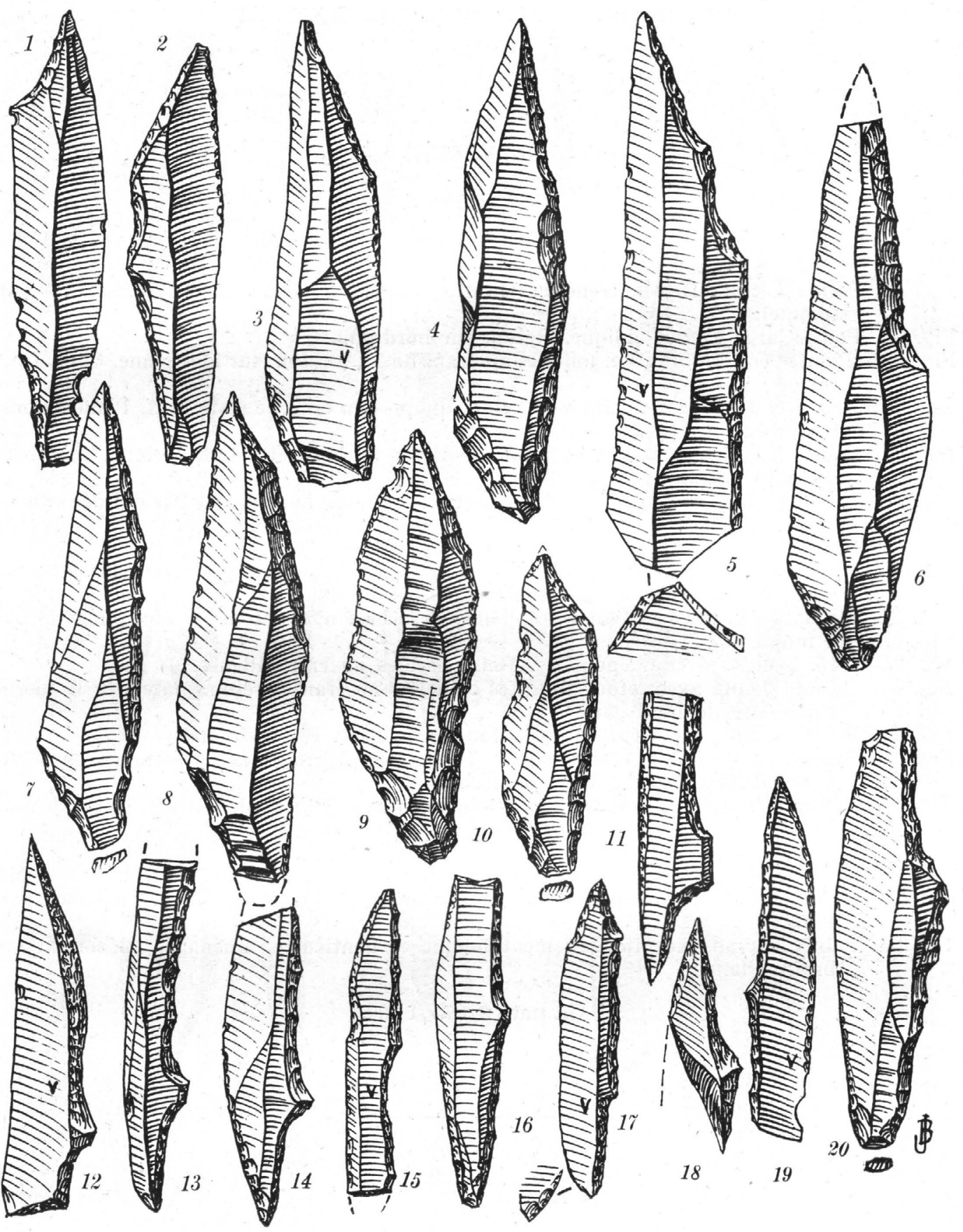

Pointes type de Châtelperron et à gibbosité.

Figure 7 - La Gravette, couche noire, dans la lignée des « Outils d'influence de l'Abri Audi et de Châtelperron », (Lacorre 1960, pl. 37, dessins J. Bouyssonie).

Figure 7 - La Gravette, black layer, in the lineage of the "tools from Abri Audi and Châtelperron" (Lacorre 1960, pl. 37, drawing J. Bouyssonie). 
Lors du colloque de l'UISPP de Nice, en $1976 \mathrm{H}$. Delporte poursuit son raisonnement : « il n'est pas possible de conserver le système périgordien et nécessaire par contre de distinguer le Castelperronien et le Gravettien (...) ; la considération d'un procédé technique, l'abattage de l'arête, ne nous semble en effet pas suffisante pour justifier le maintien d'une unité culturelle » (Delporte 1982 - p. 101).

\section{6 - Synthèse}

\section{La fin du Périgordien}

La fin du Périgordien fut graduelle, par l'abandon de la stricte contemporanéité de 1933 pour l'évolution non synchrone de 1946, les Périgordiens I, II et III, IV, V pour les Périgordiens inférieur et supérieur en 1957, puis ancien et récent. Mais progressive ne signifie certainement pas linéaire. Les changements d'opinion d'H. Delporte entre 1954, 1957 et 1969 constituent un bon exemple.

Cette proposition ne connut pas un succès immédiat et la tradition maintint l'usage des termes plus longtemps peutêtre que le modèle ne perdura réellement. Le terme est utilisé jusqu'à la fin des années 1990 (Buisson 1991; Delporte 1991 ; Eloy et Otte 1995 ; Séronie-Vivien 1995 ; Merlet 1996 ; Nespoulet 1996 ; Hans 1997 ; Soriano 1998). Difficile rétrospectivement de comprendre la diversité des points de vue probablement amalgamés sous ce terme. Difficile, voire inutile, de chercher à dater la fin du Périgordien. Formellement, le dernier des arguments de F. Bordes, l'interstratification, ne tomba que récemment (Bordes 2002). Facétie de l'histoire des fouilles, une fois encore, le Périgordien était attaqué par ce qui fût toujours son talon d'Achille : la lamelle Dufour.

Si des critiques ont été formulées à l'égard du Périgordien, ce ne fut jamais, à l'exception de $H$. Delporte, aux fondements de ce modèle. Ces reproches ne concernaient que des attributions archéologiques ponctuelles. Rien ne le renouvelait. Chaque modification se trouve donc liée à une fouille, celle de 1933 à La Ferrassie, de 1946 à Dufour, et celle de 1955 à Caminade. Lorsque D. de SonnevilleBordes remet en question le Périgordien II, c'est pour proposer l'existence de deux branches aurignaciennes. Le système est étendu, jamais remis en question.

La pérennité de l'idée d'H. Breuil (1913) d'une évolution de la pointe de Châtelperron vers la pointe de La Gravette paraît même étonnante. Elle demeurait toujours pour les derniers partisans, le meilleur argument en faveur du Périgordien. Les propositions de D. Peyrony, pour une part inspirées de la pensée breuillienne, furent systématiquement reproduites par les acteurs successifs.

Le développement des disciplines connexes, comme les datations, les évolutions dans les méthodes de fouilles n'amenèrent pas les tenants du Périgordien à remettre en question leur proposition, à renégocier ce système d'interprétation. La sériation à laquelle aboutit $\mathrm{H}$. Laville (1971) est édifiante sur ce point. Ces méthodes nouvelles ne pouvaient que s'inscrire dans ce modèle.

Le Périgordien ne pouvait se renouveler, mais seulement se ramifier, se démultiplier sur le même mode. La subdivision des ensembles devient sa condition de perduration. Nous avons pu démontrer les contre- arguments possibles à plusieurs étapes de l'histoire du Périgordien. Ces contre-arguments n'ont pourtant pas été mobilisés. Cela coïncide avec un discours d'une grande âpreté entre acteurs du Périgordien.

\section{La fin du Gravettien ?}

Ce lent délitement de l'intérieur se rejoue actuellement autour du Gravettien. Les études technologiques n'en finissent pas de démontrer l'extrême hétérogénéité de ce taxon (Pesesse 2017), comme D. Peyrony remarquait celle du Périgordien au regard de l'Aurignacien. Les réponses sont semblables : subdivision et régionalisation (De la Peña Alonso 2012). Pourtant, les études technologiques n'arrivent pas à se défaire de ce taxon, tout en y incorporant des significations très différentes (Pesesse 2017). L'évidence du Gravettien semble indépassable alors qu'il se situe parmi les derniers arrivés de la nomenclature. C'est en héritière des paradigmes en vigueur durant la phase typologique, que la technologie lithique se développe sans se situer - ou plutôt en refusant de se situer - dans cette lignée (De la Peña Alonso 2012). Si pour certains le programme historiciste serait enfin dépassé (Bradtmöller et al. 2015), cela n'est vraisemblablement pas le cas pour l'ensemble des chercheurs travaillant sur cette tranche chronologique. Et même si la période aurait à nouveau remplacé la culture, elle enferme tout autant chercheurs et paradigmes ensemble, dans des unités séparées et partiellement étanches. Le Périgordien a eu, lui, l'élégance de disparaître.

L'un des effets du Périgordien est d'avoir oblitéré toute possibilité d'analyser les liens éventuels entre Aurignacien et Gravettien. De très rares publications abordent cette question en France jusqu'à une date récente (Laplace 1966 ; Cheynier 1959 ; Delporte 1968, 1972). Ceci explique en partie le sous-investissement de ce sujet. Cette différenciation essentielle entre ces deux périodes a certainement nourri l'hypothèse d'une origine nécessairement intrusive du Gravettien, s'il ne venait pas du Périgordien inférieur (Otte 1981 ; Djindjian et Bosselin 1994).

\section{Qu'est-ce que le Périgordien ?}

Qu'est-ce que le Périgordien : une théorie, un modèle, une hypothèse ? D'un point de vue lexical, la proposition de D. Peyrony n'a jamais été présentée comme une hypothèse, mais comme un taxon archéologique qui s'est imposé au même titre que l'Aurignacien quelques années plus tôt. A. Cheynier le décrit comme une théorie (Cheynier 1963), F. Bordes également (Bordes et Labrot 1967) sans donner le même sens à ce mot. Les acteurs du Périgordien n'ont pas nécessairement précisé le statut de cet objet. S'il s'agit bien d'un taxon, lui sont rattachés plusieurs modèles interprétatifs (évolution polyphylétique, buissonnement, différentes manières de concilier biologie et culture). Le Périgordien est donc un objet hybride. Tous les taxons le sont certainement, mais pas de la même manière dans la mesure où une part des paradigmes diffère entre eux (modernité, culture pan-européenne, émergence du chasseur, parenthèse avant l'apogée, essor de l'individu, réponse au changement climatique).

Le Périgordien est tout à la fois un cadre de perception de la continuité, une manière singulière de mettre en relation 
convergence technique et stratigraphie. Le Périgordien est autant un mode de subdivision chronologique et géographique qui cherche en morcelant ces deux entités à reconstruire de la continuité. L'histoire complexe du Périgordien pourrait donc se comprendre à travers un schéma simple. Nous pourrions dire que le Périgordien a horreur du vide et ce vide interprétatif, naît au moment où se rompt le fil qui lie a priori succession stratigraphique et continuité technique. Ce modèle se développe pour redonner une nécessaire logique évolutive à des données qui défient le mode de rationalité des préhistoriens, au fil des fouilles. Cette idée de continuité ne pouvant être infléchie, les stratigraphies devront alors se recomposer.

La divergence entre $\mathrm{H}$. Breuil et $\mathrm{D}$. Peyrony n'est peut-être pas aussi franche et plusieurs correspondances peuvent être faites entre ces deux raisonnements. La pensée de $H$. Breuil ne peut être réduite à une approche unilinéaire, dans la mesure où il envisage pour le Paléolithique inférieur l'existence de deux phyla (Lippé 2012), sans oublier les évolutions parallèles de la séquence européenne du Paléolithique supérieur (Breuil 1913). La reconnaissance de faciès durant le Moustérien (Peyrony 1921) alimente certainement cette vision des choses, comme en atteste le succès ultérieur de ce terme pour les ensembles attribués à présent au Gravettien.

Il faudrait également vérifier que $\mathrm{H}$. Breuil et $\mathrm{D}$. Peyrony ont bien la même manière de penser la stratigraphie. La succession stratigraphique vaudrait succession chronologique relative pour $\mathrm{H}$. Breuil. Pour D. Peyrony, la succession stratigraphique pourrait dans certains cas avoir une valeur chronologique stricte : deux ensembles différents sus-jacents à un ensemble identique peuvent être contemporains. Un important travail reste donc à accomplir pour comprendre la relation entre ces deux points de vue.

\section{Le Périgordien, quelle erreur ?}

Qu'est-ce que le Périgordien : une erreur, une illusion collective ? Le Périgordien ne peut se départir de cette connotation, presque mythologie, d'une genèse de l'homme placée sous les combats répétés des CombeCapelle et des Cro-Magnon, qui parfois ont su sympathiser et échanger certaines techniques, aux marges de la Dordogne. Cela participe largement à la relégation du Périgordien, comme une branche morte ou déviante dans un arbre généalogique prestigieux. Pourtant, il semble peu opérant de considérer le Périgordien comme une erreur, par la suite rattrapée pour revenir au modèle de $\mathrm{H}$. Breuil, les noms de l'Aurignacien une fois changés. Ce qui suit le Périgordien n'est pas le retour à l'Aurignacien. Tous ces chercheurs ne se sont pas trompés pendant plusieurs décennies, aveuglés par un dogme.

Plusieurs possibilités s'offrent alors à nous. Soit le Périgordien est une illusion collective, une erreur d'un demisiècle, dans ce cas nous pouvons garder en l'état notre mode d'objectivation. Soit considérer que le Périgordien avait la même légitimité scientifique qu'un taxon actuel, au regard du « principe de symétrie » de D. Bloor, dans ce cas interroger notre mode d'objectivation. Si l'on écrit l'histoire du point de vue des gagnants, effectivement, il n'est pas nécessaire de s'intéresser au Périgordien. II suffit pour les praticiens d'une discipline de ramener une période antérieure de la recherche à un état d'erreur pour rendre autoréalisatrice sa propre conception du progrès scientifique. Mais pourquoi les mêmes sites ne livrent-ils pas les mêmes stratigraphies ? La stratigraphie de La Ferrassie n'existe-t-elle pas ? Pourquoi l'interprétation de la lamelle Dufour n'a pas réussi à se stabiliser durant cette période? Ces formulations reprennent l'idée de substances pré-existantes qui seraient révélées par la fouille et l'analyse, car c'est vraisemblablement la perspective dans laquelle se situaient ces chercheurs. Pour aborder ces données sans les reléguer à un état scientifique primitif, une perspective inspirée de l'anthropologie symétrique (Latour 1991) est appropriée, sans intégrer pour autant l'ensemble du dispositif analytique déployé par cet auteur (Latour 2001 pour la méthodologie et les définitions des termes qui seront utilisés, dont le glossaire p. 323 - 333).

\section{Des collectifs hybrides}

Présentée du point de vue des archéologues, cette histoire confine à un rôle passif les principaux acteurs de cet événement. Les sites et les vestiges attendraient patiemment les chercheurs pour retranscrire objectivement leur sens univoque. Pourtant, sites et objets génèrent et organisent ces débats, sans agir selon les mêmes modalités en fonction des circonstances. Deux exemples peuvent être pris : La Ferrassie et la lamelle Dufour.

Le Grand Abri de La Ferrassie polarise largement l'activité archéologique au début du $X X X^{e}$ siècle, de manière discontinue. II intervient dans les débats à plusieurs reprises et pour des raisons diverses (comme la préparation d'un colloque par exemple). Les conclusions qu'il contribue à faire advenir sont très diverses :

- en 1905 - 1907 : La Ferrassie participe à la reconnaissance de l'Aurignacien pré-solutréen par H. Breuil, D. Peyrony et L. Capitan ;

- en 1922 : La Ferrassie maintient en accord D. Peyrony et H. Breuil ;

- en 1932 : La Ferrassie génère un désaccord entre eux ; le site donne à D. Peyrony les argument pour construire le Périgordien ;

- en 1969 : La Ferrassie permettra à H. Delporte de penser le Gravettien.

La stratigraphie de La Ferrassie a ainsi contribué à faire émerger l'Aurignacien, le Périgordien et le Gravettien. Depuis H. Delporte, la stratigraphie du site a encore changé (Texier 2006).

Comme évoqué plus haut, à l'exception de Delporte (1954), le Périgordien n'est jamais interrogé sur ses fondements ; la critique provient toujours d'un site archéologique. Elle implique que de nouvelles découvertes ou de nouvelles fouilles sont les plus à même de modifier notre compréhension du passé. Ce mode de raisonnement scientifique accorde donc une place plus importante aux sites qu'à l'analyse des paradigmes. Le site devient donc l'acteur central des propositions qui seront effectuées. Dans le cadre du Périgordien, il y a donc une analogie entre le processus de fouille et le processus de découverte du passé. Nul besoin de mobiliser les cadres interprétatifs pour repenser les problèmes, la fouille suffit. Du point de vue de ceux qui partagent cette idée de la découverte, des renouvellements, perçus comme le progrès scientifique, s'opèrent effectivement. L'intérêt du Périgordien est de situer dans quelles circonstances peuvent s'opérer ces renouvellements. En l'occurrence, cette possibilité est reversée aux sites archéologiques et donc à l'aléa de la découverte. 
Cette pratique est constituée par une différence sujet/objet. Le sujet historique révèle les caractères du site, lui-même anhistorique ou selon une modalité qui serait d'un ordre strictement naturel. Très loin de ce « rêve empiriste » (Tilley 1989), la fouille, plutôt que la découverte, la révélation d'un monde naturel par un sujet historique pourrait donc plutôt être perçue comme un " événement 》 (Latour 2001 qui emprunte ce terme à Whitehead) ce qui rétablit la relation entre humains et non-humains en les replaçant dans une trame historique commune. Le collectif constitué par la fouille mêle de manière intime acteurs humains et nonhumains comme des pré-conceptions sur l'évolution technique, des rapports entre personnes, des outils et méthodes de fouille et le site lui-même dans ses différentes dimensions. La fouille produit à la fois le site et le fouilleur (Yarrow 2003).

Si le Périgordien est largement associé à l'idée de filiation entre les industries à pointes à dos abrupts, on a pu constater l'importance de la lamelle Dufour dans son histoire. Dans ce cas, ce sont les objets et la catégorie à laquelle ces lamelles sont assignées qui vont agir. Elles tiennent alors plusieurs rôles ;

- en 1922, à La Ferrassie, elles vont permettre à D. Peyrony d'envisager la discontinuité dans le changement technique. - en 1938, à Dufour, leur découverte parfait l'idée de rupture profonde entre Aurignaciens inférieur et moyen, c'est-à-dire entre Périgordien I et Aurignacien 1. A posteriori, Dufour mélange les gisements de Pair-non-Pair et Bos-del-Ser où ces lamelles étaient associées à d'autres catégories d'objets du Moustérien et du Périgordien I ;

- en 1948, elles unifient l'Aurignacien sur un vaste espace géographique et construisent des ponts par l'Autriche et la Crimée jusqu'au Proche-Orient ;

- en 1955, à Caminade, paradoxalement, elles permettent de renforcer le Périgordien en lui ôtant son maillon le plus faible : le Périgordien II. Simultanément, elles transfèrent le modèle Périgordien à l'Aurignacien.

Tour à tour, en fonction des fouilles, ces objets unifient l'Aurignacien, le décomposent en phyla, le subdivisent en périodes (Demars et Laurent 1989 ; Chiotti 2003 ; Michel 2010). Après G. Laplace (1966), ces lamelles sont oubliées pendant une quarantaine d'années par certains membres de la communauté scientifique, mais pas le phylum méditerranéen (Bazile 1976 ; Onoratini 1986), pour être réinventées au début des années 2000 (Bon 2002 ; Bordes 2005). À présent pour certains auteurs, elles contribuent à reconsidérer le point de départ du Paléolithique supérieur (Bordes et Teyssandier 2011). Dans certains cas, ces lamelles retentissent longtemps après leur découverte. À Quinçay, deux décennies après la fin des fouilles, ces lamelles rétablissent le lien entre Châtelperronien et Aurignacien (Roussel 2014) défait par D. Peyrony en 1922.

Si l'on s'intéresse à l'utilisation de la lamelle Dufour (O'Farrell 2005 ; Pelegrin et O'Farrell 2005 ; Normand et al. 2008 ; Porraz et al. 2010 ; Pasquini 2013), on néglige peutêtre parmi ses fonctions la diversité des propositions qu'elle contribue à générer. Difficile ou plutôt peu fécond, de voir en cela la succession erronée des interprétations avant de parvenir à ce qui serait aujourd'hui la compréhension de la lamelle Dufour. La question ne porte pas non plus sur une éventuelle polysémie de l'objet. Ce n'est pas le nombre de sens qui est en jeux mais la possibilité que les collectifs qui intègrent ces objets parviennent à stabiliser une explication qui rendrait compte exclusivement de la supposée nature de l'objet (Latour 1991).

Dans les exemples évoqués précédemment, il y a donc plusieurs lamelles Dufour puisque les objets d'une même catégorie ont des propriétés différentes voire même contradictoires : unir ou diviser des espaces, réagencer la chronologie, intégrer ou exclure le Châtelperronien, modifier le commencement du Paléolithique supérieur ... Quelle est la bonne lamelle Dufour? La question ne peut se présenter ainsi.

Ces variations d'interprétation sont fonction du collectif organisé par et autour de ces objets. Leur compréhension est relationnelle et ne renvoie pas à une essence préexistante (Shanks 1998 ; Holtorf 2002). Tous les éléments du réseau intégrant ces objets ne sont pas pour autant agencés de manière aléatoire dans la mesure où ces agencements sont eux-mêmes " historicisés 》 (Latour 2001). Les objets et catégories d'attribution participent d'ailleurs activement à la stabilisation des énoncés. Alors que ces différents préhistoriens énoncent les faits comme s'ils révélaient la substance de l'objet ; l'objet paraît plutôt être « substancialisé » (Latour 2001) durant ces processus. Actuellement la grande lamelle Dufour n'existe pas sans la modernité. Chaque analyse d'un site du Protoaurignacien reconduit et étend cette relation, plus qu'elle ne la démontre. Cette modernité est ainsi reconnue, constatée, observée objectivement dans chaque ensemble étudié. Comme pour le Périgordien, les objets incorporent des concepts, des idées qui pourront ainsi acquérir une certaine longévité. Cela renvoie à une propriété générale des objets qui ne saurait être valable que dans le passé. Comme la modernité n'est pas déduite des assemblages mais projetée sur eux, en eux (Pesesse sous presse), elle est donc théoriquement à même de disparaître. Dans ce cas, de nouvelles fouilles ne sont donc pas nécessaires pour abandonner ce paradigme.

C'est justement dans ce cadre que la démarche est reproductible, non en fonction d'une adéquation entre l'objet et une supposée réalité passée. Dans ce cas, la reproduction ne valide pas pour autant la proposition mais s'explique d'abord par l'extension du collectif qui déploie son raisonnement (Latour 1991). En ce sens, le Périgordien - comme tout taxon actuellement - pouvait être reconnu dans plusieurs sites. Observer l'interstratification (Bordes et Labrot 1967) ne tient pas uniquement à un problème géoarchéologique ou une lecture orientée par une visite antérieure au Piage (Bordes 2002).

Pour quitter une narration linéaire produite du point de vue anthropocentré et androcentré, cette histoire peut être perçue à travers différentes perspectives, si l'on reconnaît un caractère d'actant aux non-humains. Ils contribuent à organiser périodiquement des collectifs qui vont générer des observations reproductibles. Toutes ces propositions ne peuvent pas être ramenées sur un seul système d'évaluation. II semble donc bien plus fécond de considérer et d'analyser la variété de ces collectifs plutôt que de les classer en fonction d'un critère supérieur : l'existence d'un mode unique d'objectivation ou la réalité passée (Shanks et Tilley 1992). L'objectif serait de considérer avec joie la difficulté d'établir un savoir cumulatif dans notre discipline, pour tout ce que cela permet de penser. Ces collectifs deviennent alors autant de champs d'investigations à partir 
desquels on pourrait assumer les différences de positionnements qui composent déjà notre discipline, derrière une objectivité de forme. Une perspective constructiviste serait alors l'une des conditions possibles pour engager sereinement un dialogue et ne pas aboutir à une scission théorique entre deux positions caricaturales (Moro Abadia 2017).

Oublier le Périgordien, c'est-à-dire le considérer comme un épisode erroné et révolu, est bien commode. Dès lors, il suffit de l'interpréter en fonction de son contexte social, intellectuel ou historique. La discontinuité des industries a été observée parce que cette notion préexisterait aux observations de D Peyrony. On reprend la distinction entre le contenant et le contenu (Latour 2001). Pourtant à partir des sources utilisées, je n'ai aucun moyen de savoir s'il existait un lien entre la discontinuité formalisée depuis longtemps en biologie et ce que pense $\mathrm{D}$. Peyrony lorsqu'il parle des différences morphologiques entre les objets qu'il trouve à La Ferrassie. De même faire coïncider rétrospectivement certains termes comme « transformation 》 ou « perfectionnement » (Peyrony 1909) avec des théories pleinement constituées poserait de nombreux problèmes. Cette proposition mettrait en relation des entités différentes, sans documenter la manière dont cette relation a été

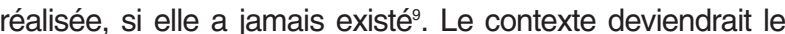
facteur agissant. J. Sackett est attentif à ne pas surinterpréter a posteriori ce modèle : "Also deserving comment is Straus's contention that Peyrony's seriation was based upon an «a priori theory of technological change» and constituted a «cultural evolutionary scheme» (1987a:I57). I find it difficult to see much here or elsewhere in Peyrony's thinking that is evolutionary at all beyond the everyday and fairly ambiguous sense with which the term is still used by prehistorians " (Sackett 1991). Cette proposition a l'avantage d'être la plus parcimonieuse. Ramenée à l'ensemble des acteurs, avec de nécessaires variations interindividuelles, elle expliquerait en partie le consensus sur le Périgordien et sa persistance. La force de conviction produite par les idées de sens commun a déjà été amplement démontrée (Stoczkowski 2001). Ainsi voir la formulation d'une classification sur des fondements raciaux et son succès comme la conséquence du contexte chronologique, ne laisse aucune capacité d'agir aux individus. Cela ne constitue pas une explication suffisante. Envisager que la manière dont ces scientifiques ont envisagé la relation entre vestiges lithiques et osseux humains a participé à la stabilisation de ces idées semble plus opérant car démontrable dans ses modalités. Les énoncés produisent alors le contexte.

Même si l'on peut proposer des ressorts interprétatifs quant au cheminement de D. Peyrony, il est important de ne pas constituer a posteriori le Périgordien comme une entité stable, homogène et ainsi l'essentialiser. Le Périgordien de D. Peyrony n'est certainement pas celui d'H. Delporte. D. Peyrony a réalisé un grand nombre de fouilles et a ainsi procédé à de nombreuses attributions de gisements au Périgordien. II a constamment intégré les fouilles antérieures et contemporaines réalisées par d'autres fouilleurs à cette classification. Le succès de ce modèle ne réside pas dans l'adhésion des acteurs à un modèle théorique général, mais de manière très pragmatique à une pratique située, routinière de l'attribution des sites, l'interprétation étant reversée aux vestiges. Les objets contiendraient leur propre signification, révélée par l'analyse. Dans ce cas, leur proximité morphologique traduirait directement leur proximité phylétique.

À ce stade, le parallèle entre approches typologiques et technologiques devient trop évident pour ne pas l'évoquer. Le statut de l'objet - sa valeur de témoin objectif - n'a pas été révoqué avec ce changement de méthode. Cet aspect a certainement contribué au transfert de certains paradigmes associés à l'outillage, en dépit du changement méthodologique (De la Peña Alonso 2012). Cette conviction en l'objectivité de l'objet ne permet pas à la controverse d'investir des aspects plus structuraux de la démarche, en particulier les cadres interprétatifs. Comme pour le Périgordien, la description objective des artefacts, de nouvelles fouilles suffisent amplement pour penser le changement et les relations entre ensembles lithiques. Après 50 ans d'études technologiques, il serait peut-être temps de reconsidérer la confiance que nous accordons aux objets.

\section{Remerciements}

Merci à Cédric Sarrazin pour sa traduction en anglais.

\section{Références bibliographiques}

BAZILE F. 1976 - Datations absolues sur les niveaux paléolithiques supérieurs anciens de la grotte de l'Esquicho Grapaou (Sainte-Anastasie, Gard), Bulletin de la Société Préhistorique Française, 73, p. 205-207.

BERNALDO DE QUIROS F., NEIRA A. 2012 - Evolucion del termino "Gravetiense ", Pensando el Gravetiense: nuevos datos para la región cantábrica en su contexto peninsular y pirenaico, C. de las Heras Martín, J. A. Lasheras Corruchaga, Á. Arrizabalaga Valbuena, M. de la Rasilla Vives (ed. lit.), p. 24-32.

BLOOR D. 1976 - Knowledge and Social Imagery, chap. 1, Chicago, University of Chicago Press.

BON F. 2002 - L'Aurignacien entre mer et océan. Réflexion sur l'unité des phases anciennes de l'Aurignacien dans le sud de la France, Paris, Société préhistorique française (coll. Mémoire, 29), $253 \mathrm{p}$.

BORDES F. 1958 - Nouvelles fouilles à Laugerie-Haute Est. Premiers résultats, L'Anthropologie, 62, p. 205-244.

BORDES F., LABROT J. 1967 - La stratigraphie du gisement de Roc de Combe (Lot) et ses implications, Bulletin de la Société Préhistorique Française, 64, p. 15 à 28.

BORDES F., SONNEVILLE-BORDES S. 1966 Protomagdalénien ou Périgordien VII, L'Anthropologie, 70, p. 113-122.

(9) Par exemple, dans le même numéro que la publication du Ruth en 1909, un article de E. Rabaud porte sur Lamarck, mais s'il le connaissait qu'en pensait D. Peyrony? 
BORDES J.-G. 2002 - Les interstratifications Châtelperronien/Aurignacien du Roc-de-Combe et du Piage (Lot, France). Analyse taphonomique des industries lithiques ; conséquences archéologiques. Mémoire de Doctorat. Université de Bordeaux I.

BORDES J.-G. 2005 - La séquence aurignacienne du Nord de l'Aquitaine : variabilité des productions lamellaires à Caminade-Est, Roc-de-Combe, le Piage et CorbiacVignoble II, In : Le Brun-Ricalens F. (Ed.), Productions lamellaires attribuées à l'Aurignacien : Chaînes opératoires et perspectives techno-culturelles. XIV ${ }^{e}$ congrès de I'UISPP, Liège (2-8 septembre 2001). Luxembourg : ArchéoLogiques $n^{\circ} 1$, p. 123-154.

BORDES J.-G., TEYSSANDIER N. 2011 - The Upper paleolithic nature of the Châtelperronian in South-Western France : archaeostratigraphic and lithic evidence, Quaternary International, 246 -1-2, 382 - 388.

BOSSELIN B., DJINDJIAN F. 1994 - La chronologie du Gravettien français, Préhistoire européenne, 6, p. 77-115.

BOUYSSONIE J. 1923 - Station préhistorique de Bos-delSer près Brive (Corrèze), Association Française pour l'Avancement des Sciences, $47^{\circ}$ session, Bordeaux, p. 617-622)

BOUYSSONIE J. 1944 - La Grotte Dufour, près Brive (Corrèze), Bulletin de la Société Préhistorique française, 41-10, 186-192.

BRADTMÖLLER M., ARRIZABALAGA A., CALVO A., IRIARTE CHIAPUSSO M. J. \& PEÑA P. de la 2015 - From Upper Perigordian to the current Non hierarchical Gravettian in the Cantabrian Region (Northern Spain): Recent changes, current challenges. In: S. Sázelová, M. Novák and A. Mizerová (eds.). Forgotten times and spaces: New perspectives in paleoanthropological, paleoetnological and archeological studies. Brno: Institute of Archeology of the Czech Academy of Sciences; Masaryk University, 245257.

BREUIL H. 1905 - Essai de stratigraphie des dépôts de l'âge du Renne, Congrès Préhistorique de France, Périgueux, p. 74 à 83.

BREUIL H. 1907a - Les gisements Présolutréens du type d'Aurignac. Coup d'œil sur le plus ancien âge du Renne, Congrès International d'Anthropologie et d'Archéologie Préhistoriques, $13^{\mathrm{e}}$ session, Monaco, 1906, p. 323-350.

BREUIL H. 1907b - La question aurignacienne, Etude critique de stratigraphie comparée, Revue Préhistorique.

BREUIL H. 1913 - Les subdivisions du Paléolithique supérieur et leur signification, Congrès International d'Anthropologie et d'Archéologie Préhistoriques, $\mathrm{XIV}^{\mathrm{e}}$ session, 1912, Genève.

BREUIL H. 1937 - Les subdivisions du Paléolithique supérieur et leur signification, Congrès International d'Anthropologie et d'Archéologie Préhistoriques, $\mathrm{XIV}^{\mathrm{e}}$ session, Genève, $2^{\mathrm{e}}$ édition.

BUISSON D. 1991 - Le Périgordien du Blot (Cerzat, HauteLoire), Bulletin de la Société Préhistorique Française, 88, p. 104 à 108.
CAPITAN L., PEYRONY D. 1905 - Fouilles à la Ferrassie (Dordogne), Congrès Préhistorique de France, Périgueux, p. 143-144.

CAPITAN L., PEYRONY D. 1907 - Les fouilles de la Ferrassie (Dordogne). Contribution à l'étude de l'Aurignacien, Congrès Préhistorique de France, $3^{\circ}$ session, Autun, p. 186 à 188.

CAPITAN L., PEYRONY D. 1920 - Une nouvelle fouille à la Ferrassie, Association Française pour l'Avancement des Sciences, $44^{\mathrm{e}}$ session, Strasbourg, p. 540-542.

CARTAILHAC E. 1907 - Le moustiérien et le pré-solutréen ou aurignacien des Grottes de Grimaldi, Congrès Internationale d'Anthropologie et d'Archéologie Préhistoriques, $13^{\circ}$ session, Monaco, 1906, p. 135-154.

CHAMPAGNE F., ESPITALIE R. 1967 - La stratigraphie du Piage, Note préliminaire, Bulletin de la Société Préhistorique Française, 64, p. 29 à 34.

CHEYNIER A. 1953 - Les lamelles à bord abattu. Autonomie. Origine. Evolution et Usages possibles, Bulletin de la Société Préhistorique Française, 51, p. 81 à 85.

CHEYNIER A. 1955 - Lettre ouverte à M. Angelroth, suite à sa publication sur le Périgordien et l'Aurignacien en Belgique, Bulletin de la société Préhistorique Française, 52, p. 515-517.

CHEYNIER A. 1959 - Note pour servir à l'étude des niveaux Inter Aurignaco-Gravétiens, Congrès Préhistorique de France, $\mathrm{XVl}^{\mathrm{e}}$ session, Principauté de Monaco, 28 août au 5 septembre 1959, p. 365-384.

CHEYNIER A. 1960 - Place pour le Gravétien, Bulletin de la Société Préhistorique Française, 57, p. 389 à 414.

CHEYNIER A. 1963 - Le Périgordien n'est qu'une « théorie », Bulletin de la société Préhistorique Française, 90, p. 23-26.

CHEYNIER A., DALEAU F. 1963 - La caverne de Pairnon-Pair (Gironde), Société archéologique de Bordeaux, Documents d'Aquitaine, $220 \mathrm{p}$.

CHIOTTI L. 2003 - Les productions lamellaires dans l'Aurignacien de l'abri Pataud, Les Eyzies-de-Tayac (Dordogne), Gallia préhistoire, 45, p. 113-156.

COMBIER J. 1955 - Observations complémentaires sur le Périgordien, Bulletin de la société Préhistorique Française, 52, p. 602 à 603.

COMBIER J., MONTET-WHITE A. 2002 - Solutré 1968 1998, Mémoire de la Société préhistorique de France, $\mathrm{n}^{\circ} 30$.

COYE N. 2011 - Une discipline en reconstruction : la préhistoire française de la première moitié du $X X^{e}$ siècle, Le Genre humain, vol. 50, n. 1, p. 199-220.

DANIEL R. 1952 - Procès verbal de la séance du 18 décembre 1952, $\mathrm{n}^{\circ} 10$, Bulletin de la Société Préhistorique Française, 49, p. 620. 
DELPORTE H. 1953 - Périgordien de Fontenioux, Bulletin de la société Préhistorique Française, 50, p. 9-11.

DELPORTE H. 1954 - Le Périgordien, Bulletin jubilaire de la Société Préhistorique Française, 51, 44 à 48.

DELPORTE H. 1957a - Corrézien ou Brivien, Bulletin de la société Préhistorique Française, 54, p. 43-44.

DELPORTE H. 1957b - L'industrie périgordienne de l'Abri du Facteur ou de la Forêt, à Tursac (Dordogne), Bulletin de la Société d'Etudes et de Recherches Préhistoriques, $\mathrm{n}^{\circ} 7$, 24-37.

DELPORTE H. 1968 - L'abri du Facteur à Tursac, Gallia Préhistoire, 11, p. 1-112.

DELPORTE H. 1969 - Les fouilles du Musée des Antiquités Nationales à la Ferrassie, Bulletin des Antiquités Nationales, p. 15-28.

DELPORTE H. 1972 - L'Aurignacien et le Bayacien de la Gravette : Mise en oeuvre statistique et problèmes posés, Bulletin de la Société Préhistorique Française, t. 69, Etudes et travaux, fasc. 1, p. 337-346.

DELPORTE H. 1982 - L'organisation du Périgordien supérieur en France, et ses rapports avec le Périgordien d'Europe occidentale, in : Aurignacien et Gravettien en Europe, Actes des réunions de la $10^{\circ}$ Commission de I'UISPP, « Aurignacien-Périgordien-Gravettien et cultures dérivées », Cracovie-Nitra, ERAUL n ${ }^{\circ}$, Liège, p. 83 à 106.

DELPORTE H. 1991 - La séquence aurignacienne et périgordienne sur la base des travaux récents réalisés en Périgord, Bulletin de la Société Préhistorique Française, 88 p. 243-256.

DEMARS P.-Y., LAURENT P. 1989 - Types d'outils lithiques du Paléolithique supérieur en Europe, Cahiers du Quaternaire, 14, Paris, Presses du CNRS.

DJINDJIAN F., BOSSELIN B. 1994 - Périgordien et Gravettien : l'épilogue d'une contradiction ?, Préhistoire européenne, 6, p. 117-131.

DUBOIS S. 2006 - La « bataille aurignacienne ». Genèse d'un débat d'école dans son contexte historique et intellectuel, Mémoire de DEA, Université de Toulouse Le Mirail, 59 p.

DUBOIS S., BON F. 2006 - « Henri Breuil et les origines de la bataille aurignacienne ", in N. Coye (dir.), Sur les chemins de la préhistoire. L'abbé Breuil du Périgord à l'Afrique du Sud, Paris, Somogy, p. 135-147.

ELOY L., OTTE M. 1995 - Le Périgordien de l'abri sousroche de Goyet (Namur, Belgique), Bulletin des Chercheurs de la Wallonie, 35, p. 25-40

GARROD D. A. E. 1936 - The Upper Palaeolithic in the light of recent discoveries. Nature, 826-830.

GOUTAS N. 2004 - Caractérisation et évolution du Gravettien en France par l'approche techno-économique des industries en matières dures animales (étude de six gisements du Sud-Ouest), Mémoire de Doctorat, Université de Paris I, Panthéon-Sorbonne, 675 p.
GROENEN M. 1994 - Pour une histoire de la Préhistoire, Grenoble, Jérôme Million, 603 p.

GUILLOMET-MALMASSARI V. 2012 - D'une révolution à l'autre. Pour une épistémologie de la problématique de transition en Préhistoire, Mémoire LIV, Société Préhistorique Française, 132 p.

HANS J.-M. 1997 - Périgordien à burins de Noailles: le site de Hautmougey (Vosges, canton de Bains-les-Bains), Bulletin de la Société Préhistorique luxembourgeoise, p. 55 à 66 .

HOLTORF C. 2002 - Notes on the history life of a pot sherd, Journal of Material Culture, 7-1, 49-71.

KLARIC L. 2003 - L'unité technique des industries à burins du Raysse dans leur contexte diachronique. Réflexions sur la diversité culturelle au Gravettien à partir des données de la Picardie, d'Arcy-sur-Cure, de Brassempouy et du Cirque de la Patrie, Paris, Université de Paris I - Panthéon Sorbonne, Thèse de doctorat, $426 \mathrm{p}$.

LACORRE F. 1933a - Les armatures de flèches de la Gravette, $\mathrm{XV}^{\circ}$ Congrès International Anthropologie et d'Archéologie Préhistorique de Paris, 20 à 27 septembre 1931, p. 409 à 415.

LACORRE F. 1933b - Les armatures de flèches de la Gravette. Niveau et industries auxquelles elles se rattachent. Procès-verbaux de la Société linéenne de Bordeaux, séance du 21 juin 1933, p. 78 à 87.

LACORRE F. 1956 - Sur le Périgordien II, Bulletin de la société Préhistorique Française, 53, p. 140-143.

LACORRE F. 1959a - Tableau chronologique du Périgordien et de l'Aurignacien et de leurs complexes, Bulletin de la Société Préhistorique française, 55, p. 53 à 57.

LACORRE F. 1959b - Tableau chronologique du Périgordien et de l'Aurignacien (suite) Classification des industries à éclats et lames à bord abattu, Bulletin de la Société Préhistorique française, 55, p. 433 - 440.

LACORRE F. 1960 - La Gravette, le Gravétien et le Bayacien, Laval, Imprimerie Barneoud.

LAPLACE G. 1966 - Recherches sur l'origine et l'évolution des complexes leptolithiques, Ecole française de Rome, Mélanges d'Archéologie et d'Histoire, Suppléments, 4, Paris, De Boccard, 1 vol., 586 p.

LATOUR B. 1991 - Nous n'avons jamais été modernes. Essai d'anthropologie symétrique, Paris, La Découverte, Poche / Sciences humaines et sociales, 207 p.

LATOUR B. 2001 - L'espoir de Pandore. Pour une version réaliste de l'activité scientifique. Trad. de l'anglais $\mathrm{D}$. Gille, Paris La Découverte, 347 p.

LAVILLE H. 1971 - Sur la contemporanéité du Périgordien et de l'Aurignacien : la contribution du géologue, Bulletin de la Société Préhistorique Française, 68, p. 171-174.

LIPPÉ R. 2012 - Nature, culture et progrès. Histoire comparative du concept de transition entre paléolithiques 
moyen et supérieur, Mémoire de Doctorat, Université de Montréal, Université de Bordeaux I, 296 p.

MERLET J.-C. 1996 - Le Périgordien supérieur et l'Azilien d'Arcet à Montaut (Landes), Archéologie des Pyrénées occidentales et des Landes, 105, p. 119-126.

MICHEL A. 2010 - L'Aurignacien récent (post-ancien) dans le Sud-Ouest de la France : variabilité des productions lithiques. Révision taphonomique et techno-économique des sites de Caminade-Est, abri Pataud, Roc-de-Combe, Le Flageolet I, La Ferrassie et Combemenue, thèse de doctorat, université Bordeaux I, 600 p.

MORO ABADIA O. 2017 - Bridging the gap in archaeological theory: an alternative account of scientific 'progress' in archaeology, World Archaeology, 49-2, 271-280.

NESPOULET R. 1996 - Le Périgordien VI de l'abri Pataud, les Eyzies-de-Tayac, Dordogne. Etude technologique et typologique de l'industrie lithique de la couche 3. Mémoire de doctorat du Muséum d'Histoire Naturelle.

NORMAND C., O'FARRELL M., RIOS GARAIZAR J. 2008 - Quelles(s) utilisation(s) pour les productions lamellaires de l'Aurignacien archaïque ? Quelques données et réflexions à partir des exemplaires de la grotte d'Isturitz (Pyrénées-Atlantiques ; France), In : J.-M. Pétillon, M.-H. Dias-Meirinho, P. Cattelain, M. Honegger, C. Normand, N. Valdeyron (Eds.), Recherches sur les armatures de projectiles du Paléolithique supérieur au Néolithique, Actes du colloque $\mathrm{C} 83, \mathrm{XV}^{\mathrm{e}}$ congrès de I'UISPP, Lisbonne, 4-9 septembre 2006, Palethnologie, 1, p. 7-46.

O'FARRELL M. 2005 - Etude préliminaire des éléments d'armature lithique de l'Aurignacien ancien de Brassempouy, In : F. Le Brun-Ricalens, F. Bon, J.-G. Bordes (Eds), Productions lamellaires attribuées à l'Aurignacien : chaînes opératoires et perspectives technoculturelles. $\quad \mathrm{XV}^{\mathrm{e}}$ congrès de I'U.I.S.P.P. Liège (2-8 septembre 2001), ArchéoLogiques, n¹, p. 395-412.

ONORATINI G. 1986 - Découverte en Provence orientale (grotte Rainaude) d'une industrie souche de l'Aurignacien. Cette civilisation est-elle monolithique ?, Bulletin de la Société Préhistorique Française, 83, 240-256.

OTTE M. 1981 - Le Gravettien en Europe centrale, Dissertationes Archaeologicae Gandenses, vol XX, De Tempel, Brugge.

PASQUINI A. 2013 - Les traces de notre passé européen. Le Protoaurignacien au début du Paléolithique supérieur: l'éclairage de la tracéologie. Thèse de doctorat de l'université de Aix-Marseille, $146 \mathrm{p}$.

PELEGRIN J., O'FARRELL M. 2005 - Les lamelles retouchées ou utilisées de Castanet. In: Lebrun-Ricalens, F. (Ed.), Productions lamellaires attribuées à l'Aurignacien: chaînes opératoires et perspectives technoculturelles, ArchéoLogiques 1. Musée National d'Histoire et d'Art, Luxembourg, p. 103-122.

PEÑA ALONSO P. de la 2009 - Revisión crítica de los conjuntos líticos gravetienses y su contexto arqueológico en la Península Ibérica, Complutum, 20(1), p.29-53.

PEÑA ALONSO P. de la 2012 - A proposito del Gravetiense, El paso del cultura a tecnocomplejo, Complutum, 23(1), 41-62.

PESESSE D. 2008 - Place du Bayacien dans la structuration du Gravettien, Gallia, 50, 23-44.

PESESSE D. 2013 - Les premières sociétés gravettiennes, Analyse comparée des systèmes lithiques de la fin de l'Aurignacien aux débuts du Gravettien, Paris, CTHS, $285 \mathrm{p}$.

PESESSE D. 2017 - Is it still appropriate to talk about the Gravettian ? Datas from lithic industries in Western Europe, Quartär, 64, 107-128.

PESESSE D., sous presse, Une relation ambiguë entre sujets et objets, l'analyse de silex taillés durant le paléolithique supérieur, Techniques \& Culture, Technographie.

PEYRONY D. 1909 - Station préhistorique du Ruth près le Moustiers (Dordogne), Aurignacien, Solutréen et Magdalénien, Revue de l'Ecole d'Anthropologie de Paris, $156-176$.

PEYRONY D. 1921 - Le Moustérien, ses faciès, Association Française pour l'Avancement des Sciences, $44^{\circ}$ session, Strasbourg, 1920, p. 496-497.

PEYRONY D. 1922 - Nouvelles observations sur le Moustérien final et l'Aurignacien inférieur, Association française pour l'Avancement des Sciences, $46^{\mathrm{e}}$ congrès, Montpellier, 1922, 511-514.

PEYRONY D. 1932 - Paléolithiques supérieurs européen et africain. Rapports entre eux, Revue anthropologique, 42, p. 126-141.

PEYRONY D. 1933 - Les industries aurignaciennes dans le bassin de la Vézère, Bulletin de la Société Préhistorique Française, 30, p. 543 à 559.

PEYRONY D. 1936 - Le Périgordien et l'Aurignacien (Nouvelles observations), Bulletin de la Société Préhistorique Française, 33, p. 616 à 619.

PEYRONY D., PEYRONY E. 1938 - Laugerie-Haute, près des Eyzies (Dordogne), Archives de l'Institut de Paléontologie Humaine, Mémoire 19.

PEYRONY D. 1939 - Sur l'importance de l'étude géologique des gisements préhistoriques, Revue Anthropologique, p. 54-62.

PEYRONY D. 1940 - Les Grimaldiens en Périgord, L'Anthropologie, 49, p. 702-708.

PEYRONY D. 1946 - Une mise au point au sujet de l'aurignacien et du Périgordien, Bulletin de la Société Préhistorique française, 42-43, p. 232-237.

PEYRONY D. 1948 - Le Périgordien, l'Aurignacien et le Solutréen en Eurasie, d'après les dernières fouilles, Bulletin de la Société Préhistorique Française, 45, p. 305-328. 
PORRAZ G., SIMON P., PASQUINI A. 2010 - Identité technique et comportements économiques des groupes proto-aurignaciens : la grotte de l'Observatoire (principauté de Monaco). Gallia préhistoire, 52, p. 33-59.

PRADEL L. 1952a - Caractéristiques lithiques des différents niveaux périgordiens en France, Bulletin de la Société Préhistorique Française, 49, p. 531 à 543.

PRADEL L. 1952b - La grotte périgordienne et aurignacienne du Fontenioux, commune de Saint-Pierrede-Maillé (Vienne), Bulletin de la Société Préhistorique Française, 49, p. 413 à 432.

PRADEL L. 1953 - Considérations sur le Périgordien de la grotte du Fontenioux, Bulletin de la Société Préhistorique Française, 50, p. 422 à 426.

PRADEL L. 1955 - Périgordien et Aurignacien, Constatations, possibilités et apparences, Bulletin de la Société Préhistorique Française, 52, p. 604 à 607.

PRADEL L. 1956 - Périgordien, Corrézien, Aurignacien, Bulletin de la Société Préhistorique Française, 53, p. 462.

PRADEL L. 1961 - Sur le synchronisme du Périgordien et de l'Aurignacien, Bulletin de la Société Préhistorique Française, 65, p. 621 à 627.

ROUSSEL M. 2014 - Des lamelles retouchées au Châtelperronien : diffusion d'idées entre derniers Néandertaliens et premiers Hommes modernes migrants In: M. Otte \& F. Lebrun-Ricalens (dir.), «Modes de contacts et de déplacements au Paléolithique eurasiatique», Actes du Colloque international de la commission 8 (Paléolithique supérieur) de l'UISPP, Université de Liège, 28-31 mai 2012 : 491-510, Sep 25, 2014.

RUTOT A. 1907 - Le Présolutréen ou Aurignacien en Belgique, Congrès Préhistorique de France, III, Autun, p. $179-181$.

SACKETT J. R. 1991 - Straight archaeology French style: The phylogenetic paradigm in historic perspective. In Perspectives on the Past, edited by G. A. Clark, p. 109-139. University of Pennsylvania Press, Philadelphia.

SERONIE-VIVIEN M.-R. 1995 - La grotte de Pégourié, Caniac-du-Causse (Lot); Périgordien-Badegoulien-AzilienAge du Bronze, Préhistoire quercinoise, supplément $\mathrm{n}^{\circ} 2$.

SHANKS M. 1998 - The Life of an Artefact in an Interpretive Archaeology, Fennoscandia Archaeologica, 15, 15-42.

SHANKS M. S., TILLEY C. 1992 - Re-constructing archaeology, Theory and practice, Second Edition, Routledge, $287 \mathrm{p}$.

SONNEVILLE-BORDES D. de 1952 - Esquisse d'une évolution typologique du Paléolithique supérieur en Périgord. Défense et illustration de la méthode statistique, L'Anthropologie, 58, p. 197 à 230.
SONNEVILLE-BORDES D. de 1955a - La question du Périgordien II, Bulletin de la société Préhistorique Française, 52, p. 187-201.

SONNEVILLE-BORDES D. de 1955b - A propos du Périgordien, Bulletin de la société Préhistorique Française, 52, p. 597 à 601.

SONNEVILLE-BORDES D. de 1955c - La grotte de Chanlat et la question du Périgordien II, L'Anthropologie, 59, p. 357 à 360.

SONNEVILLE-BORDES D. 1960 - Le Paléolithique supérieur en Périgord, Bordeaux, Delmas, 2 vol., 558 p.

SORIANO S. 1998 - Microgravettes du Périgordien de Rabier à Lanquais (Dordogne), Analyse technologique et fonctionnelle, Gallia Préhistoire, CNRS, Paris, 40, 75-94.

SOULIER P. 2007 - Un siècle de Bulletin de la société préhistorique française (1904-2004) : déambulation bibliographique au cœur d'une société, savante et centenaire, In : Un siècle de construction du discours scientifique en Préhistoire, "Des idées d'hier ... », J. Evin dir., Congrès du centenaire de la Société préhistorique française, Avignon, 21 au 25 septembre 2004, $\mathrm{XXVl}^{\mathrm{e}}$ Congrès Préhistorique de France, vol. 1. p. 27-125.

STOCZKOWSKI W. 2001 - Anthropologie naïve, Anthropologie savante. De l'origine de l'Homme, de l'imagination et des idées reçues. Cnrs Editions, Empreintes de l'homme, $256 \mathrm{p}$.

TEXIER J.-P. 2006 - La Ferrassie, In Texier J.P. (dir.) Livretguide de l'excursion de la Société géologique française "Sites préhistoriques du Périgord », Pôle international de Préhistoire, p. 22-29.

TILLEY C. 1989 - Excavation as theatre, Antiquity, 63, 275-280.

TRAVERS M. 1981 - Sur quelques questions de terminologie scientifique - La Banque des mots, 21-4, p. 3-18.

TRONEL J. 2015 - André Cheynier, Un éminent préhistorien détenu au camp de Mauzac, Esprit de Pays.

WHITE R., ROUSSOT A. 2003 - Résumé de ma vie : une note autobiographique de Denis Peyrony, Bulletin de la Société Archéologique du Périgord, 80, 453 - 472.

YARROW T. 2003 - Artefactual Persons: The Relational Capacities of Persons and Things in the Practice of Excavation, Norwegian Archaeological Review, 36:1, 65-73, 
